Supporting Information

Table S1. ${ }^{13} \mathrm{C}$ experimental NMR shifts for $\mathbf{2} \mathbf{a}-\mathbf{c}^{\mathbf{2 +}}$ and $\mathbf{3 b}, \mathbf{c}^{\mathbf{2 + a}}$

Table S2.Calculated ${ }^{13} \mathrm{C}$ NMR shifts for $\mathbf{2 a - d ^ { 2 + }}$ and $\mathbf{3 a -} \mathbf{c}^{\mathbf{2 +}}$

Table S3. Ratio of calculated and experimental ${ }^{1} \mathrm{H}$ shifts.

Table S4. Cartesian Coordinates and total energies for singlets and triplets of $\mathbf{2}^{\mathbf{2 +}}$ and $\mathbf{3}^{\mathbf{2 +}}$

Table S5. HOMO and LUMO energies for $\mathbf{2}^{\mathbf{2 +}}, \mathbf{3}^{\mathbf{3 +}}$

Spectrum S1. 3,6 Dimethyl tetrabenzo[5.5]fulvalene, $\mathbf{3 a}^{2+},{ }^{1} \mathrm{H}$ NMR.

Spectrum S2. 3,6 Dichloro tetrabenzo[5.5]fulvalene, $3 \mathbf{b}^{2+},{ }^{1} \mathrm{H}$ NMR.

Spectrum S3. 3,6 Dichloro tetrabenzo[5.5]fulvalene, $\mathbf{3} \mathbf{b}^{2+},{ }^{13} \mathrm{C}$ NMR.

Spectrum S4. 3,6-Difluorotetrabenzo[5.5]fulvalene, $3 \mathbf{c}^{2+},{ }^{1} \mathrm{H}$ NMR.

Spectrum S5. 3,6-Difluorotetrabenzo[5.5]fulvalene, $3 \mathbf{c}^{2+},{ }^{13} \mathrm{C}$ NMR.

Spectrum S6. 3,6 Dimethyl tetrabenzo[5.5]fulvalene, 3a, ${ }^{1} \mathrm{H}$ NMR.

Spectrum S7. 3,6 Dimethyl tetrabenzo[5.5]fulvalene, 3a, ${ }^{13} \mathrm{C}$ NMR.

Spectrum S8. 3,6 Dichloro tetrabenzo[5.5]fulvalene, 3b, ${ }^{1} \mathrm{H}$ NMR.

Spectrum S9. 3,6 Dichloro tetrabenzo[5.5]fulvalene, 3b, ${ }^{13} \mathrm{C}$ NMR.

Spectrum S10. 3,6-Difluorotetrabenzo[5.5]fulvalene, 3c, ${ }^{1} \mathrm{H}$ NMR.

Spectrum S11. 3,6-Difluorotetrabenzo[5.5]fulvalene, 3c, ${ }^{13} \mathrm{C}$ NMR.

Synthesis of $\mathrm{SO}_{2} \mathrm{ClF}$. 
Table S1. ${ }^{13} \mathrm{C}$ experimental NMR shifts for $\mathbf{2} \mathbf{a}-\mathbf{c}^{2+}$ and $\mathbf{3 b}, \mathbf{c}^{\mathbf{2 + a}}$

\begin{tabular}{|l|l|l|l|l|r|r|}
\hline Compound & $\mathbf{2 a}^{2+}$ & $\mathbf{2 b}^{2+}$ & $\mathbf{2 c}^{2+}$ & $\mathbf{2 d}^{\mathbf{2 +}}$ & $\mathbf{3 b}^{2+}$ & $\mathbf{3 c}^{2+}$ \\
\hline 1,8 & 145.2 & 144.6 & 132.0 & & 145.3 & 144.9 \\
\hline 2,7 & 145.1 & 137.0 & 167.6 & & 142.1 & 122.3 \\
\hline 3,6 & 159.5 & 162.2 & 145.5 & & 167.4 & 182.6 \\
\hline 4,5 & 131.0 & 131.9 & 132.4 & & 130.6 & 121.3 \\
\hline 9 & 189.6 & $193.0^{b}$ & $193.1^{c}$ & & 188.6 & 182.6 \\
\hline 10,13 & 148.3 & 146.6 & 146.3 & & 152.3 & 141.1 \\
\hline 11,12 & 152.4 & 149.0 & 150.2 & & 154.8 & 152.7 \\
\hline $1^{\prime}, 8^{\prime}$ & 149.2 & 143.5 & 145.0 & & 144.3 & 145.6 \\
\hline $2^{\prime}, 7^{\prime}$ & 135.7 & 132.7 & 137.0 & & 135.0 & 136.3 \\
\hline $3^{\prime}, 6^{\prime}$ & 158.5 & 159.0 & 160.1 & & 158.6 & 159.4 \\
\hline $4^{\prime}, 5^{\prime}$ & 129.9 & 131.2 & 131.2 & & 142.0 & 130.9 \\
\hline $9^{\prime}$ & 188.6 & $186.8^{b}$ & $186.0^{c}$ & & 180.9 & 188.7 \\
\hline $10^{\prime}, 13^{\prime}$ & 148.1 & 145.3 & 146.1 & & 150.0 & 146.6 \\
\hline $11^{\prime}, 12^{\prime}$ & 152.1 & 153.1 & 153.1 & & 156.5 & 153.2 \\
\hline $\mathrm{CH}_{3}$ & 17.4 & & & & & \\
\hline
\end{tabular}

${ }^{a}$ Solvent, $0.75 \mathrm{~g}$ of $\mathrm{SbF}_{5}, 1.5 \mathrm{~mL}$ of $\mathrm{SO}_{2} \mathrm{ClF}$; temperature, $-50^{\circ} \mathrm{C}$, reference, TMS oin acetone- $d_{6}$ in an external capillary. ${ }^{b}$ These assignments could be interchanged. ${ }^{c}$ These assignments could be interchanged.

Table S2.Calculated ${ }^{13} \mathrm{C}$ NMR shifts for $\mathbf{2 a - \mathbf { d } ^ { 2 + }}$ and $\mathbf{3 a}-\mathbf{c}^{2+}$

\begin{tabular}{|l|r|r|r|r|r|r|r|}
\hline Compound & $\mathbf{2 a}^{\mathbf{2 +}}$ & $\mathbf{2 b}^{\mathbf{2 +}}$ & $\mathbf{2 c}^{\mathbf{2 +}}$ & $\mathbf{2 d}^{\mathbf{2 +}}$ & $\mathbf{3 a}^{2+}$ & $\mathbf{3 b}^{2+}$ & $\mathbf{3 c}^{\mathbf{2 +}}$ \\
\hline 1,8 & 133.5 & 133.7 & 121.9 & & 127.5 & 126.4 & 131.5 \\
\hline 2,7 & 140.7 & 144.0 & 157.8 & & 124.7 & 124.5 & 112.3 \\
\hline 3,6 & 153.3 & 153.9 & 141.8 & & 170.3 & 170.3 & 173.1 \\
\hline 4,5 & 121.9 & 123.2 & 123.9 & & 122.5 & 121.0 & 111.8 \\
\hline 9 & 179.3 & 184.5 & 184.6 & & 169.1 & 165.2 & 168.2 \\
\hline 10,13 & 138.8 & 141.7 & 141.5 & & 131.6 & 129.8 & 128.5 \\
\hline 11,12 & 154.8 & 145.4 & 141.4 & & 138.8 & 142.0 & \\
\hline $1^{\prime}, 8^{\prime}$ & 130.7 & 131.3 & 131.5 & 132 & 130.7 & 131.2 & 131.5 \\
\hline $2^{\prime}, 7^{\prime}$ & 125.2 & 126.1 & 126.2 & 126 & 124.9 & 125.2 & 125.5 \\
\hline $3^{\prime}, 6^{\prime}$ & 150.4 & 152.0 & 152.1 & 151 & 149.8 & 150.1 & 151.0 \\
\hline $4^{\prime}, 5^{\prime}$ & 120.4 & 121.4 & 121.5 & 121 & 120.3 & 120.8 & 121.1 \\
\hline $9^{\prime}$ & 178.5 & 174.7 & 174.6 & 178 & 179.9 & 178.8 & 178.2 \\
\hline $10^{\prime}, 13^{\prime}$ & 133.4 & 134.3 & 134.5 & 134 & 133.3 & 133.7 & 133.9 \\
\hline $11^{\prime}, 12^{\prime}$ & 142.0 & 142.6 & 142.8 & 143 & 142.1 & 142.4 & 142.5 \\
\hline $\mathrm{CH}_{3}$ & 12.7 & & & & 20.5 & & \\
\hline
\end{tabular}


Table S3. Ratio of calculated and experimental ${ }^{1} \mathrm{H}$ shifts.

\begin{tabular}{|l|r|r|r|r|r|r|}
\hline & \multicolumn{1}{|l|}{$\mathbf{2 a}^{2+}$} & \multicolumn{1}{l|}{$\mathbf{2 b}^{2+}$} & $\mathbf{2 c}^{2+}$ & $\mathbf{3 a}^{2+}$ & $\mathbf{3 b}^{2+}$ & $\mathbf{3}^{2+}$ \\
\hline $\mathrm{H}_{1}, \mathrm{H}_{8}$ & 1.06 & 1.31 & 1.19 & 0.92 & 0.93 & 0.86 \\
\hline $\mathrm{H}_{2}, \mathrm{H}_{7}$ & & & & 0.94 & 0.91 & 0.95 \\
\hline $\mathrm{H}_{3}, \mathrm{H}_{6}$ & 0.98 & 0.95 & 0.90 & & & \\
\hline $\mathrm{H}_{4}, \mathrm{H}_{5}$ & 1.03 & 1.61 & 1.12 & 0.93 & 0.92 & 0.98 \\
\hline $\mathrm{H}_{1}, \mathrm{H}_{8}$, & 0.93 & 0.94 & 0.88 & 0.93 & 0.94 & 0.95 \\
\hline $\mathrm{H}_{2}, \mathrm{H}_{7}$, & 0.91 & 0.92 & 0.85 & 0.90 & 0.90 & 0.91 \\
\hline $\mathrm{H}_{3^{\prime}}, \mathrm{H}_{6}$, & 0.90 & 0.91 & 0.86 & 0.89 & 0.89 & 0.90 \\
\hline $\mathrm{H}_{4}, \mathrm{H}_{5}$, & 0.93 & 0.96 & 0.90 & 0.92 & 0.91 & 0.92 \\
\hline $\mathrm{CH}_{3}$ & 0.60 & & & 0.52 & & \\
\hline Ave-fl & 0.96 & 1.05 & 0.96 & 0.92 & 0.91 & 0.92 \\
\hline St dv & 0.06 & 0.22 & 0.13 & 0.02 & 0.02 & 0.04 \\
\hline
\end{tabular}

Table S4. Cartesian Coordinates and total energies for singlets and triplets of $\mathbf{2}^{2+}$ and $\mathbf{3}^{\mathbf{2 +}}$ $2 \mathbf{a}^{2+}$

Singlet

Total energy, -1078.453611 hartrees
C $\quad-0.349999 \quad-0.000574 \quad 0.885986$
$\begin{array}{llll}\text { C } & -0.349999 & -0.000574 & -0.574540\end{array}$
$\begin{array}{llll}\text { C } & -0.324605 & 1.167870 & -1.403642\end{array}$
C $\quad-0.375393-1.169018-1.403642$
C $\quad-0.336723 \quad 2.534983-1.062833$
C $\quad-0.363275-2.536131-1.062833$
$\begin{array}{llll}\text { C } & -0.339392 & 3.503598 & -2.078156\end{array}$
$\begin{array}{llll}\text { C } & -0.360606 & -3.504746 & -2.078156\end{array}$
$\begin{array}{llll}\text { C } & -0.342036 & 3.055047 & -3.409644\end{array}$
$\begin{array}{llll}\text { C } & -0.357962 & -3.056195 & -3.409644\end{array}$
$\begin{array}{llll}\text { C } & -0.343984 & 1.680390 & -3.768978\end{array}$
$\begin{array}{llll}\text { C } & -0.356014 & -1.681538 & -3.768978\end{array}$
$\begin{array}{llll}\text { C } & -0.341698 & 0.739969 & -2.767779\end{array}$
$\begin{array}{llll}\text { C } & -0.358300 & -0.741117 & -2.767779\end{array}$
$\begin{array}{llll}\text { C } & 0.684795 & 0.539475 & 1.716854\end{array}$
$\begin{array}{llll}\text { C } & -1.384793 & -0.540623 & 1.716854\end{array}$
$\begin{array}{llll}\text { C } & 1.914515 & 1.135624 & 1.377375\end{array}$
$\begin{array}{llll}\text { C } & -2.614513 & -1.136772 & 1.377375\end{array}$
$\begin{array}{llll}\text { C } & 2.759550 & 1.549766 & 2.410798\end{array}$
$\begin{array}{llll}\text { C } & -3.459548 & -1.550914 & 2.410798\end{array}$
$\begin{array}{llll}\text { C } & 2.382189 & 1.357925 & 3.741871\end{array}$
$\begin{array}{llll}\text { C } & -3.082187 & -1.359073 & 3.741871\end{array}$
$\begin{array}{llll}\text { C } & 1.147627 & 0.743986 & 4.093966\end{array}$
$\begin{array}{llll}\text { C } & -1.847625 & -0.745134 & 4.093966\end{array}$
$\begin{array}{llll}\text { C } & 0.310907 & 0.334994 & 3.084083\end{array}$
$\begin{array}{llll}\text { C } & -1.010905 & -0.336142 & 3.084083 \mathrm{~V}\end{array}$ 


$\begin{array}{cccc}\mathrm{H} & -0.349999 & 2.848047 & -0.022609 \\ \mathrm{H} & -0.349999 & -2.849195 & -0.022609 \\ \mathrm{H} & -0.350030 & 3.793075 & -4.207856 \\ \mathrm{H} & -0.349968 & -3.794223 & -4.207856 \\ \mathrm{H} & -0.349643 & 1.403736 & -4.818839 \\ \mathrm{H} & -0.350355 & -1.404884 & -4.818839 \\ \mathrm{H} & 2.207447 & 1.269320 & 0.340586 \\ \mathrm{H} & -2.907445 & -1.270468 & 0.340586 \\ \mathrm{H} & 3.712916 & 2.012988 & 2.180650 \\ \mathrm{H} & -4.412914 & -2.014136 & 2.180650 \\ \mathrm{H} & 3.049969 & 1.679658 & 4.535753 \\ \mathrm{H} & -3.749967 & -1.680806 & 4.535753 \\ \mathrm{H} & 0.894416 & 0.610001 & 5.141062 \\ \mathrm{H} & -1.594414 & -0.611149 & 5.141062 \\ \mathrm{C} & -0.345142 & 4.978512 & -1.762842 \\ \mathrm{H} & -0.422267 & 5.159821 & -0.687734 \\ \mathrm{H} & 0.572178 & 5.459276 & -2.121802 \\ \mathrm{H} & -1.186723 & 5.481406 & -2.251297 \\ \mathrm{C} & -0.354856 & -4.979660 & -1.762842 \\ \mathrm{H} & -0.277731 & -5.160969 & -0.687734 \\ \mathrm{H} & -1.272176 & -5.460424 & -2.121802 \\ \mathrm{H} & 0.486725 & -5.482554 & -2.251297\end{array}$

Triplet

Total energy, -1078.44813941 hartrees

$\begin{array}{llll}\text { C } & 0.000000 & 0.000000 & 0.915826\end{array}$

$\begin{array}{llll}\text { C } & 0.000000 & 0.000000 & -0.516526\end{array}$

$\begin{array}{llll}\text { C } & 0.052228 & 1.165911 & -1.385459\end{array}$

C $\quad-0.052228 \quad-1.165911 \quad-1.385459$

$\begin{array}{llll}\text { C } & 0.021211 & 2.525877 & -1.102398\end{array}$

$\begin{array}{llll}\text { C } & -0.021211 & -2.525877 & -1.102398\end{array}$

$\begin{array}{llll}\text { C } & 0.001307 & 3.461637 & -2.162854\end{array}$

$\begin{array}{lllll}\text { C } & -0.001307 & -3.461637 & -2.162854\end{array}$

$\begin{array}{llll}\text { C } & 0.006435 & 2.993091 & -3.512264\end{array}$

$\begin{array}{lllll}\text { C } & -0.006435 & -2.993091 & -3.512264\end{array}$

$\begin{array}{llll}\text { C } & 0.009696 & 1.642748 & -3.818142\end{array}$

$\begin{array}{lllll}\text { C } & -0.009696 & -1.642748 & -3.818142\end{array}$

$\begin{array}{lllll}\text { C } & 0.020115 & 0.712760 & -2.761913\end{array}$

$\begin{array}{lllll}\text { C } & -0.020115 & -0.712760 & -2.761913\end{array}$

$\begin{array}{llll}\text { C } & 0.887850 & 0.764764 & 1.766904\end{array}$

$\begin{array}{llll}\text { C } & -0.887850 & -0.764764 & 1.766904\end{array}$

$\begin{array}{llll}\text { C } & 1.996552 & 1.571040 & 1.452107\end{array}$

$\begin{array}{llll}\text { C } & -1.996552 & -1.571040 & 1.452107\end{array}$

$\begin{array}{llll}\text { C } & 2.745260 & 2.122761 & 2.494905\end{array}$

$\begin{array}{llll}\text { C } & -2.745260 & -2.122761 & 2.494905\end{array}$

$\begin{array}{llll}\text { C } & 2.405835 & 1.853327 & 3.823190\end{array}$ 


\begin{tabular}{|c|c|c|c|}
\hline & -2.405835 & -1.853327 & 23190 \\
\hline $\mathrm{C}$ & 1.315986 & 1.010514 & 4.151711 \\
\hline $\mathrm{C}$ & -1.315986 & -1.010514 & 4.151711 \\
\hline $\mathrm{C}$ & 0.570958 & 0.468499 & 3.126882 \\
\hline$C$ & -0.570958 & -0.468499 & 3.126882 \\
\hline $\mathrm{H}$ & 0.000000 & 2.887470 & -0.079753 \\
\hline $\mathrm{H}$ & 0.000000 & -2.887470 & -0.079753 \\
\hline $\mathrm{H}$ & -0.007241 & 3.724874 & -4.314852 \\
\hline $\mathrm{H}$ & 0.007241 & -3.724874 & -4.314852 \\
\hline $\mathrm{H}$ & -0.006263 & 1.314255 & -4.852678 \\
\hline $\mathrm{H}$ & 0.006263 & -1.314255 & -4.852678 \\
\hline $\mathrm{H}$ & 2.283544 & 1.757904 & 0.422474 \\
\hline $\mathrm{H}$ & -2.283544 & -1.757904 & 0.422474 \\
\hline $\mathrm{H}$ & 3.600901 & 2.751701 & 2.273344 \\
\hline $\mathrm{H}$ & -3.600901 & -2.751701 & 2.273344 \\
\hline $\mathrm{H}$ & 2.998964 & 2.283882 & 4.624431 \\
\hline $\mathrm{H}$ & -2.998964 & -2.283882 & 4.624431 \\
\hline $\mathrm{H}$ & 1.093981 & 0.797481 & 5.192805 \\
\hline $\mathrm{H}$ & -1.093981 & -0.797481 & 5.192805 \\
\hline 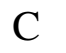 & -0.037867 & 713 & -1.85 \\
\hline $\mathrm{H}$ & -0.020361 & 5.161517 & -0.826946 \\
\hline $\mathrm{H}$ & 0.810685 & 5.435300 & -2.376974 \\
\hline $\mathrm{H}$ & -0.942800 & 5.376799 & -2.330756 \\
\hline C & 0.037867 & -4.931713 & -1.894167 \\
\hline$U$ & 0.020361 & -5.161517 & -0.826946 \\
\hline $\mathrm{H}$ & -0.810685 & -5.435300 & -2.376974 \\
\hline & 0.942800 & -5.376799 & -2.330756 \\
\hline \multicolumn{4}{|c|}{$2 \mathbf{b}^{2+}$} \\
\hline \multicolumn{4}{|c|}{ Singlet } \\
\hline \multicolumn{4}{|c|}{ Total energy, -1918.98139 hartrees } \\
\hline $\mathrm{C}$ & -0.070096 & 0.132598 & 1.023389 \\
\hline $\mathrm{C}$ & -0.070096 & 0.132598 & -0.437607 \\
\hline $\mathrm{C}$ & -0.045988 & 1.299016 & -1.270945 \\
\hline $\mathrm{C}$ & -0.094204 & -1.033820 & -1.270945 \\
\hline $\mathrm{C}$ & -0.055808 & 2.661416 & -0.922137 \\
\hline $\mathrm{C}$ & -0.084384 & -2.396220 & -0.922137 \\
\hline $\mathrm{C}$ & -0.053632 & 3.606578 & -1.956456 \\
\hline $\mathrm{C}$ & -0.086560 & -3.341382 & -1.956456 \\
\hline $\mathrm{C}$ & -0.056395 & 3.187859 & -3.292803 \\
\hline $\mathrm{C}$ & -0.083797 & -2.922663 & -3.292803 \\
\hline $\mathrm{C}$ & -0.061800 & 1.811400 & -3.641125 \\
\hline $\mathrm{C}$ & -0.078392 & -1.546204 & -3.641125 \\
\hline $\mathrm{C}$ & -0.061644 & 0.872708 & -2.636506 \\
\hline $\mathrm{C}$ & -0.078548 & -0.607512 & -2.636506 \\
\hline $\mathrm{C}$ & 0.960879 & 0.681614 & 1.852251 \\
\hline
\end{tabular}




$\begin{array}{lrcc}\mathrm{C} & -1.101071 & -0.416418 & 1.852251 \\ \mathrm{C} & 2.186927 & 1.288999 & 1.513858 \\ \mathrm{C} & -2.327119 & -1.023803 & 1.513858 \\ \mathrm{C} & 3.025918 & 1.713272 & 2.547389 \\ \mathrm{C} & -3.166110 & -1.448076 & 2.547389 \\ \mathrm{C} & 2.648269 & 1.519504 & 3.878398 \\ \mathrm{C} & -2.788461 & -1.254308 & 3.878398 \\ \mathrm{C} & 1.418753 & 0.893255 & 4.229806 \\ \mathrm{C} & -1.558945 & -0.628059 & 4.229806 \\ \mathrm{C} & 0.587589 & 0.474050 & 3.219874 \\ \mathrm{C} & -0.727781 & -0.208854 & 3.219874 \\ \mathrm{H} & -0.070096 & 2.990237 & 0.111604 \\ \mathrm{H} & -0.070096 & -2.725041 & 0.111604 \\ \mathrm{H} & -0.059953 & 3.935936 & -4.079610 \\ \mathrm{H} & -0.080239 & -3.670740 & -4.079610 \\ \mathrm{H} & -0.067013 & 1.533829 & -4.690614 \\ \mathrm{H} & -0.073179 & -1.268633 & -4.690614 \\ \mathrm{H} & 2.482841 & 1.423563 & 0.477978 \\ \mathrm{H} & -2.623033 & -1.158367 & 0.477978 \\ \mathrm{H} & 3.974739 & 2.186008 & 2.317872 \\ \mathrm{H} & -4.114931 & -1.920812 & 2.317872 \\ \mathrm{H} & 3.311408 & 1.849260 & 4.672912 \\ \mathrm{H} & -3.451600 & -1.584064 & 4.672912 \\ \mathrm{H} & 1.165423 & 0.759404 & 5.276895 \\ \mathrm{H} & -1.305615 & -0.494208 & 5.276895 \\ \mathrm{Cl} & -0.057278 & 5.293312 & -1.574347 \\ \mathrm{Cl} & -0.082914 & -5.028116 & -1.574347\end{array}$

Triplet

Total energy, -1918.97953 hartrees

$\begin{array}{llll}\text { C } & 0.000000 & 0.000000 & 1.062679\end{array}$

$\begin{array}{llll}\text { C } & 0.000000 & 0.000000 & -0.372068\end{array}$

$\begin{array}{llll}\text { C } & 0.050616 & 1.164580 & -1.242857\end{array}$

$\begin{array}{lllll}\text { C } & -0.050616 & -1.164580 & -1.242857\end{array}$

$\begin{array}{llll}\text { C } & 0.021936 & 2.521413 & -0.952686\end{array}$

$\begin{array}{llll}\text { C } & -0.021936 & -2.521413 & -0.952686\end{array}$

$\begin{array}{llll}\text { C } & 0.007662 & 3.432297 & -2.032154\end{array}$

$\begin{array}{llll}\text { C } & -0.007662 & -3.432297 & -2.032154\end{array}$

$\begin{array}{llll}\text { C } & 0.008981 & 2.995085 & -3.384104\end{array}$

$\begin{array}{llll}\text { C } & -0.008981 & -2.995085 & -3.384104\end{array}$

$\begin{array}{llll}\text { C } & 0.009970 & 1.641043 & -3.677064\end{array}$

$\begin{array}{llll}\text { C } & -0.009970 & -1.641043 & -3.677064\end{array}$

$\begin{array}{llll}\text { C } & 0.019634 & 0.713740 & -2.618422\end{array}$

$\begin{array}{llll}\text { C } & -0.019634 & -0.713740 & -2.618422\end{array}$

$\begin{array}{llll}\text { C } & 0.892042 & 0.759602 & 1.911504\end{array}$

$\begin{array}{llll}\text { C } & -0.892042 & -0.759602 & 1.911504\end{array}$ 


$\begin{array}{llll}\mathrm{C} & 2.003410 & 1.563200 & 1.595342 \\ \mathrm{C} & -2.003410 & -1.563200 & 1.595342 \\ \mathrm{C} & 2.752189 & 2.115919 & 2.637296 \\ \mathrm{C} & -2.752189 & -2.115919 & 2.637296 \\ \mathrm{C} & 2.410687 & 1.849464 & 3.965711 \\ \mathrm{C} & -2.410687 & -1.849464 & 3.965711 \\ \mathrm{C} & 1.318130 & 1.008855 & 4.295934 \\ \mathrm{C} & -1.318130 & -1.008855 & 4.295934 \\ \mathrm{C} & 0.572936 & 0.466488 & 3.272286 \\ \mathrm{C} & -0.572936 & -0.466488 & 3.272286 \\ \mathrm{H} & 0.000000 & 2.897726 & 0.063498 \\ \mathrm{H} & 0.000000 & -2.897726 & 0.063498 \\ \mathrm{H} & -0.003072 & 3.734962 & -4.177354 \\ \mathrm{H} & 0.003072 & -3.734962 & -4.177354 \\ \mathrm{H} & -0.006290 & 1.311510 & -4.711192 \\ \mathrm{H} & 0.006290 & -1.311510 & -4.711192 \\ \mathrm{H} & 2.292810 & 1.747013 & 0.565734 \\ \mathrm{H} & -2.292810 & -1.747013 & 0.565734 \\ \mathrm{H} & 3.608737 & 2.743363 & 2.415230 \\ \mathrm{H} & -3.608737 & -2.743363 & 2.415230 \\ \mathrm{H} & 3.003594 & 2.280818 & 4.766725 \\ \mathrm{H} & -3.003594 & -2.280818 & 4.766725 \\ \mathrm{H} & 1.095279 & 0.799624 & 5.337600 \\ \mathrm{H} & -1.095279 & -0.799624 & 5.337600 \\ \mathrm{Cl} & -0.022785 & 5.109259 & -1.703341 \\ \mathrm{Cl} & 0.022785 & -5.109259 & -1.703341\end{array}$

\begin{tabular}{|c|c|c|c|}
\hline \multicolumn{4}{|c|}{$2 c^{2+}$} \\
\hline \multicolumn{4}{|c|}{ Singlet } \\
\hline \multicolumn{4}{|c|}{ Total energy, -1198.25732 hartrees } \\
\hline$C$ & 0.000000 & 0.000000 & 0.000000 \\
\hline $\mathrm{C}$ & 0.000000 & 0.000000 & 1.460943 \\
\hline $\mathrm{C}$ & 1.167204 & 0.000000 & 2.293457 \\
\hline $\mathrm{C}$ & -1.167204 & 0.000000 & 2.293457 \\
\hline $\mathrm{C}$ & 2.529472 & -0.037579 & 1.943376 \\
\hline $\mathrm{C}$ & -2.529472 & 0.037579 & 1.943376 \\
\hline $\mathrm{C}$ & 3.454521 & -0.056449 & 2.988250 \\
\hline $\mathrm{C}$ & -3.454521 & 0.056449 & 2.988250 \\
\hline $\mathrm{C}$ & 3.053562 & -0.052533 & 4.324362 \\
\hline $\mathrm{C}$ & -3.053562 & 0.052533 & 4.324362 \\
\hline $\mathrm{C}$ & 1.676812 & -0.028578 & 4.668135 \\
\hline $\mathrm{C}$ & -1.676812 & 0.028578 & 4.668135 \\
\hline $\mathrm{C}$ & 0.741473 & -0.007304 & 3.659291 \\
\hline $\mathrm{C}$ & -0.741473 & 0.007304 & 3.659291 \\
\hline $\mathrm{C}$ & 0.571130 & 1.018947 & -0.828693 \\
\hline
\end{tabular}




$\begin{array}{lrcc}\text { C } & -0.571130 & -1.018947 & -0.828693 \\ \text { C } & 1.204442 & 2.231940 & -0.490351 \\ \text { C } & -1.204442 & -2.231940 & -0.490351 \\ \text { C } & 1.646755 & 3.061530 & -1.523840 \\ \text { C } & -1.646755 & -3.061530 & -1.523840 \\ \text { C } & 1.445232 & 2.687901 & -2.854887 \\ \text { C } & -1.445232 & -2.687901 & -2.854887 \\ \text { C } & 0.792689 & 1.472034 & -3.206279 \\ \text { C } & -0.792689 & -1.472034 & -3.206279 \\ \text { C } & 0.355496 & 0.650195 & -2.196354 \\ \text { C } & -0.355496 & -0.650195 & -2.196354 \\ \text { H } & 2.878728 & -0.058023 & 0.916455 \\ \text { H } & -2.878728 & 0.058023 & 0.916455 \\ \text { H } & 3.815777 & -0.073760 & 5.097448 \\ \text { H } & -3.815777 & 0.073760 & 5.097448 \\ \text { H } & 1.394243 & -0.028931 & 5.716177 \\ \text { H } & -1.394243 & 0.028931 & 5.716177 \\ \text { H } & 1.344772 & 2.525216 & 0.545507 \\ \text { H } & -1.344772 & -2.525216 & 0.545507 \\ \text { H } & 2.139539 & 4.000129 & -1.294426 \\ \text { H } & -2.139539 & -4.000129 & -1.294426 \\ \text { H } & 1.789291 & 3.343710 & -3.649427 \\ \text { H } & -1.789291 & -3.343710 & -3.649427 \\ \text { H } & 0.653744 & 1.221523 & -4.253386 \\ \text { H } & -0.653744 & -1.221523 & -4.253386 \\ \text { F } & 4.749246 & -0.085014 & 2.697231 \\ \text { F } & -4.749246 & 0.085014 & 2.697231\end{array}$

Triplet

Total energy, -1198.2545698 hartrees

$\begin{array}{llll}\text { C } & 0.000000 & 0.000000 & 0.930356\end{array}$

$\begin{array}{llll}\text { C } & 0.000000 & 0.000000 & -0.506163\end{array}$

$\begin{array}{llll}\text { C } & 0.047737 & 1.164178 & -1.375925\end{array}$

$\begin{array}{llll}\text { C } & -0.047737 & -1.164178 & -1.375925\end{array}$

$\begin{array}{llll}\text { C } & 0.020130 & 2.520642 & -1.087000\end{array}$

$\begin{array}{llll}\text { C } & -0.020130 & -2.520642 & -1.087000\end{array}$

$\begin{array}{llll}\text { C } & 0.004803 & 3.408822 & -2.177846\end{array}$

$\begin{array}{llll}\text { C } & -0.004803 & -3.408822 & -2.177846\end{array}$

$\begin{array}{llll}\text { C } & 0.003324 & 2.992041 & -3.530537\end{array}$

$\begin{array}{lllll}\text { C } & -0.003324 & -2.992041 & -3.530537\end{array}$

$\begin{array}{llll}\text { C } & 0.006073 & 1.638852 & -3.820033\end{array}$

$\begin{array}{llll}\text { C } & -0.006073 & -1.638852 & -3.820033\end{array}$

$\begin{array}{llll}\text { C } & 0.017920 & 0.712560 & -2.755348\end{array}$

$\begin{array}{llll}\text { C } & -0.017920 & -0.712560 & -2.755348\end{array}$

$\begin{array}{llll}\text { C } & 0.893473 & 0.757631 & 1.777830\end{array}$

$\begin{array}{llll}\text { C } & -0.893473 & -0.757631 & 1.777830\end{array}$ 


\begin{tabular}{lllc} 
C & 2.005353 & 1.560961 & 1.460876 \\
$\mathrm{C}$ & -2.005353 & -1.560961 & 1.460876 \\
$\mathrm{C}$ & 2.754018 & 2.114574 & 2.502268 \\
$\mathrm{C}$ & -2.754018 & -2.114574 & 2.502268 \\
$\mathrm{C}$ & 2.411810 & 1.849358 & 3.830816 \\
$\mathrm{C}$ & -2.411810 & -1.849358 & 3.830816 \\
$\mathrm{C}$ & 1.318568 & 1.008979 & 4.162058 \\
$\mathrm{C}$ & -1.318568 & -1.008979 & 4.162058 \\
$\mathrm{C}$ & 0.573616 & 0.465904 & 3.139085 \\
$\mathrm{C}$ & -0.573616 & -0.465904 & 3.139085 \\
$\mathrm{H}$ & 0.000000 & 2.921067 & -0.079789 \\
$\mathrm{H}$ & 0.000000 & -2.921067 & -0.079789 \\
$\mathrm{H}$ & -0.011039 & 3.748181 & -4.308756 \\
$\mathrm{H}$ & 0.011039 & -3.748181 & -4.308756 \\
$\mathrm{H}$ & -0.009944 & 1.304249 & -4.852423 \\
$\mathrm{H}$ & 0.009944 & -1.304249 & -4.852423 \\
$\mathrm{H}$ & 2.295070 & 1.743871 & 0.431211 \\
$\mathrm{H}$ & -2.295070 & -1.743871 & 0.431211 \\
$\mathrm{H}$ & 3.610580 & 2.741910 & 2.280020 \\
$\mathrm{H}$ & -3.610580 & -2.741910 & 2.280020 \\
$\mathrm{H}$ & 3.004413 & 2.281570 & 4.631616 \\
$\mathrm{H}$ & -3.004413 & -2.281570 & 4.631616 \\
$\mathrm{H}$ & 1.095516 & 0.801609 & 5.204042 \\
$\mathrm{H}$ & -1.095516 & -0.801609 & 5.204042 \\
$\mathrm{~F}$ & -0.015033 & 4.698294 & -1.926218 \\
$\mathrm{~F}$ & 0.015033 & -4.698294 & -1.926218 \\
& & & \\
$\mathbf{2 d}^{2+}$ & & \multicolumn{2}{c}{} \\
$\mathrm{S}$ & & &
\end{tabular}

Singlet

Total energy, -999.809426 hartrees

$\begin{array}{lrrr}\mathrm{H} & -4.983175 & 0.987211 & 0.982431 \\ \mathrm{C} & -3.935095 & 1.187416 & 1.182101 \\ \mathrm{C} & -1.215087 & 1.793873 & 1.786477 \\ \mathrm{C} & -2.928408 & 0.525554 & 0.523334 \\ \mathrm{C} & -3.578351 & 2.163915 & 2.154461 \\ \mathrm{C} & -2.246217 & 2.462201 & 2.451727 \\ \mathrm{C} & -1.559600 & 0.826650 & 0.823336 \\ \mathrm{H} & 0.177192 & -2.020460 & 2.012547 \\ \mathrm{H} & -4.370375 & 2.690651 & 2.678765 \\ \mathrm{H} & -2.014083 & 3.212978 & 3.199285 \\ \mathrm{C} & -0.733583 & 0.000000 & 0.000000 \\ \mathrm{H} & -0.177192 & 2.020460 & 2.012547 \\ \mathrm{C} & 0.733583 & 0.000000 & 0.000000 \\ \mathrm{C} & -1.559600 & -0.826650 & -0.823336 \\ \mathrm{C} & 1.559600 & 0.826650 & -0.823336 \\ \mathrm{C} & 1.559600 & -0.826650 & 0.823336\end{array}$




$\begin{array}{cccc}\mathrm{C} & -3.578351 & -2.163915 & -2.154461 \\ \mathrm{C} & -1.215087 & -1.793873 & -1.786477 \\ \mathrm{C} & -2.928408 & -0.525554 & -0.523334 \\ \mathrm{C} & -3.935095 & -1.187416 & -1.182101 \\ \mathrm{C} & -2.246217 & -2.462201 & -2.451727 \\ \mathrm{H} & -0.177192 & -2.020460 & -2.012547 \\ \mathrm{H} & 2.014083 & -3.212978 & 3.199285 \\ \mathrm{H} & -4.983175 & -0.987211 & -0.982431 \\ \mathrm{H} & -2.014083 & -3.212978 & -3.199285 \\ \mathrm{H} & -4.370375 & -2.690651 & -2.678765 \\ \mathrm{C} & 3.578351 & 2.163915 & -2.154461 \\ \mathrm{C} & 2.928408 & 0.525554 & -0.523334 \\ \mathrm{C} & 1.215087 & 1.793873 & -1.786477 \\ \mathrm{C} & 2.246217 & 2.462201 & -2.451727 \\ \mathrm{C} & 3.935095 & 1.187416 & -1.182101 \\ \mathrm{H} & 4.983175 & -0.987211 & 0.982431 \\ \mathrm{H} & 0.177192 & 2.020460 & -2.012547 \\ \mathrm{H} & 2.014083 & 3.212978 & -3.199285 \\ \mathrm{H} & 4.983175 & 0.987211 & -0.982431 \\ \mathrm{H} & 4.370375 & 2.690651 & -2.678765 \\ \mathrm{C} & 3.578351 & -2.163915 & 2.154461 \\ \mathrm{C} & 2.928408 & -0.525554 & 0.523334 \\ \mathrm{C} & 1.215087 & -1.793873 & 1.786477 \\ \mathrm{C} & 2.246217 & -2.462201 & 2.451727 \\ \mathrm{C} & 3.935095 & -1.187416 & 1.182101 \\ \mathrm{H} & 4.370375 & -2.690651 & 2.678765\end{array}$

Triplet

Total energy, -999.7906403 hartrees

$\begin{array}{llll}\text { C } & 0.000000 & 0.000000 & 0.711403 \\ \mathrm{C} & 0.443494 & 1.083761 & 1.575918 \\ \mathrm{C} & -0.443494 & -1.083761 & 1.575918 \\ \mathrm{C} & 1.064867 & 2.300955 & 1.282981 \\ \mathrm{C} & -1.064867 & -2.300955 & 1.282981 \\ \mathrm{C} & 1.472690 & 3.120554 & 2.345444 \\ \mathrm{C} & -1.472690 & -3.120554 & 2.345444 \\ \mathrm{C} & 1.282107 & 2.724068 & 3.681845 \\ \mathrm{C} & -1.282107 & -2.724068 & 3.681845 \\ \mathrm{C} & 0.696676 & 1.488517 & 3.992196 \\ \mathrm{C} & -0.696676 & -1.488517 & 3.992196 \\ \mathrm{C} & 0.288548 & 0.665816 & 2.944932 \\ \mathrm{C} & -0.288548 & -0.665816 & 2.944932 \\ \mathrm{C} & 0.000000 & 0.000000 & -0.711403 \\ \mathrm{C} & -0.443494 & 1.083761 & -1.575918 \\ \mathrm{C} & 0.443494 & -1.083761 & -1.575918 \\ \mathrm{C} & -1.064867 & 2.300955 & -1.282981\end{array}$




\begin{tabular}{llcl}
$\mathrm{C}$ & 1.064867 & -2.300955 & -1.282981 \\
$\mathrm{C}$ & -1.472690 & 3.120554 & -2.345444 \\
$\mathrm{C}$ & 1.472690 & -3.120554 & -2.345444 \\
$\mathrm{C}$ & -1.282107 & 2.724068 & -3.681845 \\
$\mathrm{C}$ & 1.282107 & -2.724068 & -3.681845 \\
$\mathrm{C}$ & -0.696676 & 1.488517 & -3.992196 \\
$\mathrm{C}$ & \multicolumn{1}{c}{0.696676} & -1.488517 & -3.992196 \\
$\mathrm{C}$ & -0.288548 & 0.665816 & -2.944932 \\
$\mathrm{C}$ & 0.288548 & -0.665816 & -2.944932 \\
$\mathrm{H}$ & 1.245958 & 2.615397 & 0.261013 \\
$\mathrm{H}$ & -1.245958 & -2.615397 & 0.261013 \\
$\mathrm{H}$ & 1.952504 & 4.070911 & 2.134428 \\
$\mathrm{H}$ & -1.952504 & -4.070911 & 2.134428 \\
$\mathrm{H}$ & 1.610869 & 3.377953 & 4.483584 \\
$\mathrm{H}$ & -1.610869 & -3.377953 & 4.483584 \\
$\mathrm{H}$ & 0.580927 & 1.183947 & 5.027598 \\
$\mathrm{H}$ & -0.580927 & -1.183947 & 5.027598 \\
$\mathrm{H}$ & -1.245958 & 2.615397 & -0.261013 \\
$\mathrm{H}$ & 1.245958 & -2.615397 & -0.261013 \\
$\mathrm{H}$ & -1.952504 & 4.070911 & -2.134428 \\
$\mathrm{H}$ & 1.952504 & -4.070911 & -2.134428 \\
$\mathrm{H}$ & -1.610869 & 3.377953 & -4.483584 \\
$\mathrm{H}$ & 1.610869 & -3.377953 & -4.483584 \\
$\mathrm{H}$ & -0.580927 & 1.183947 & -5.027598 \\
$\mathrm{H}$ & 0.580927 & -1.183947 & -5.027598
\end{tabular}

$3 \mathbf{a}^{2+}$

Singlet

Total energy, -1078.46314 hartrees
$\begin{array}{llll}\text { C } & -0.432182 & 0.251347 & 0.006568\end{array}$
$\begin{array}{llll}\text { C } & -0.432182 & 0.251347 & 1.466967\end{array}$
$\begin{array}{llll}\text { C } & 0.734197 & 0.251347 & 2.294683\end{array}$
$\begin{array}{llll}\text { C } & -1.598562 & 0.251347 & 2.294683\end{array}$
$\begin{array}{llll}\text { C } & 2.106073 & 0.205970 & 1.972583\end{array}$
$\begin{array}{llll}\text { C } & -2.970437 & 0.296725 & 1.972583\end{array}$
$\begin{array}{llll}\text { C } & 3.035159 & 0.181483 & 3.006787\end{array}$
$\begin{array}{llll}\text { C } & -3.899523 & 0.321212 & 3.006787\end{array}$
$\begin{array}{llll}\text { C } & 2.630763 & 0.187485 & 4.356448\end{array}$
$\begin{array}{llll}\text { C } & -3.495127 & 0.315209 & 4.356448\end{array}$
$\begin{array}{llll}\text { C } & 1.234246 & 0.217648 & 4.673945\end{array}$
$\begin{array}{llll}\text { C } & -2.098610 & 0.285047 & 4.673945\end{array}$
$\begin{array}{llll}\text { C } & 0.309568 & 0.242369 & 3.661874\end{array}$
$\begin{array}{llll}\text { C } & -1.173932 & 0.260326 & 3.661874\end{array}$
$\begin{array}{llll}\text { C } & 0.157795 & 1.258257 & -0.825652\end{array}$
$\begin{array}{llll}\text { C } & -1.022159 & -0.755562 & -0.825652\end{array}$
$\begin{array}{llll}\text { C } & 0.810840 & 2.458628 & -0.487304\end{array}$ 


$\begin{array}{cccc}\mathrm{C} & -1.675204 & -1.955933 & -0.487304 \\ \mathrm{C} & 1.264917 & 3.282613 & -1.521072 \\ \mathrm{C} & -2.129281 & -2.779918 & -1.521072 \\ \mathrm{C} & 1.055478 & 2.914436 & -2.851961 \\ \mathrm{C} & -1.919842 & -2.411741 & -2.851961 \\ \mathrm{C} & 0.382884 & 1.711071 & -3.203164 \\ \mathrm{C} & -1.247248 & -1.208376 & -3.203164 \\ \mathrm{C} & -0.064663 & 0.894997 & -2.192586 \\ \mathrm{C} & -0.799701 & -0.392302 & -2.192586 \\ \mathrm{H} & 2.441007 & 0.185786 & 0.939920 \\ \mathrm{H} & -3.305371 & 0.316909 & 0.939920 \\ \mathrm{H} & 4.095070 & 0.148370 & 2.774145 \\ \mathrm{H} & 4.095070 & 0.148370 & 2.774145 \\ \mathrm{H} & -4.959434 & 0.354325 & 2.774145 \\ \mathrm{H} & 0.933869 & 0.218176 & 5.717713 \\ \mathrm{H} & -1.798233 & 0.284519 & 5.717713 \\ \mathrm{H} & 0.956943 & 2.745818 & 0.549356 \\ \mathrm{H} & -1.821307 & -2.243123 & 0.549356 \\ \mathrm{H} & 1.772956 & 4.212942 & -1.291248 \\ \mathrm{H} & -2.637320 & -3.710248 & -1.291248 \\ \mathrm{H} & 1.408715 & 3.565803 & -3.646063 \\ \mathrm{H} & -2.273079 & -3.063108 & -3.646063 \\ \mathrm{H} & 0.235200 & 1.464596 & -4.250013 \\ \mathrm{H} & -1.099564 & -0.961901 & -4.250013 \\ \mathrm{C} & 3.650639 & 0.154159 & 5.451431 \\ \mathrm{H} & 4.281720 & -0.740109 & 5.363038 \\ \mathrm{H} & 4.327267 & 1.015583 & 5.374504 \\ \mathrm{H} & 3.195704 & 0.159482 & 6.443871 \\ \mathrm{C} & -4.515003 & 0.348536 & 5.451431 \\ \mathrm{H} & -5.146084 & 1.242804 & 5.363038 \\ \mathrm{H} & -5.191631 & -0.512888 & 5.374504 \\ \mathrm{H} & -4.060068 & 0.343212 & 6.443871\end{array}$

Triplet

Total energy, -1078.4455481 hartrees

$\begin{array}{llll}\text { C } & 0.000000 & 0.000000 & 1.166144\end{array}$

$\begin{array}{llll}\text { C } & 0.000000 & 0.000000 & -0.266275\end{array}$

$\begin{array}{llll}\text { C } & 0.051001 & 1.160718 & -1.133085\end{array}$

$\begin{array}{llll}\text { C } & -0.051001 & -1.160718 & -1.133085\end{array}$

$\begin{array}{llll}\text { C } & 0.019830 & -2.530377 & -0.854473\end{array}$

$\begin{array}{llll}\text { C } & -0.000941 & 3.424473 & -1.922444\end{array}$

$\begin{array}{llll}\text { C } & 0.000941 & -3.424473 & -1.922444\end{array}$

$\begin{array}{llll}\text { C } & -0.000451 & 3.002308 & -3.295556\end{array}$

C $\quad 0.000451 \quad-3.002308 \quad-3.295556$

C $\quad 0.002615 \quad 1.632686-3.573520$

$\begin{array}{llll}\text { C } & -0.002615 & -1.632686 & -3.573520\end{array}$ 


\begin{tabular}{|c|c|c|c|}
\hline & 7408 & 6733 & +1 \\
\hline C & -0.017408 & -0.716733 & -2.518647 \\
\hline & 0.877147 & 0.777323 & 2.017678 \\
\hline & -0.877147 & -0.777323 & 2.017678 \\
\hline C & 1.975687 & 1.597842 & 1.704837 \\
\hline & -1.975687 & -1.597842 & 1.704837 \\
\hline c & 2.714888 & 2.160913 & 2.748168 \\
\hline C & -2.714888 & -2.160913 & 2.748168 \\
\hline $\mathrm{C}$ & 2.377340 & 1.887992 & 4.076333 \\
\hline $\mathrm{C}$ & -2.377340 & -1.887992 & 4.076333 \\
\hline C & 1.299969 & 1.029660 & 4.40 \\
\hline $\mathrm{C}$ & -1.299969 & -1.029660 & 4.403224 \\
\hline 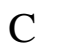 & 0.563609 & 0.477030 & 3.377416 \\
\hline $\mathrm{C}$ & -0.563609 & -0.477030 & 3.377416 \\
\hline $\mathrm{H}$ & 000 & 2.9 & 0.1 \\
\hline $\mathrm{H}$ & 0.000000 & -2.902219 & 0.163825 \\
\hline $\mathrm{H}$ & -0.028725 & 4.491031 & -1.715412 \\
\hline $\mathrm{H}$ & 0.028725 & -4.491031 & -1.715412 \\
\hline $\mathrm{H}$ & -0.018413 & 346 & 16 \\
\hline $\mathrm{H}$ & 413 & -1.2 & -4.6 \\
\hline 11 & 2.263870 & 1.787665 & 0.67 \\
\hline $\mathrm{H}$ & -2.263870 & -1.787665 & 0.675980 \\
\hline $\mathrm{H}$ & 3.561746 & 2.801810 & 2.527164 \\
\hline$U$ & 1746 & -2.801810 & 164 \\
\hline $\mathrm{H}$ & 2.962991 & 2.327768 & 4.878030 \\
\hline $\mathrm{H}$ & -2.962991 & -2.327768 & 4.878030 \\
\hline $\mathrm{H}$ & 1.079916 & 0.812677 & 5.443913 \\
\hline $\mathrm{H}$ & 16 & 677 & 913 \\
\hline $\mathrm{C}$ & -0.021312 & 4.032253 & -4.380787 \\
\hline $\mathrm{H}$ & -0.907505 & 4.674415 & -4.287765 \\
\hline $\mathrm{H}$ & 0.850307 & 4.695771 & -4.300717 \\
\hline $\mathrm{H}$ & -0.023093 & 3.582074 & -5.375475 \\
\hline $\mathrm{C}$ & 0.021312 & -4.0 & -4.3 \\
\hline $\mathrm{H}$ & 0.907505 & -4.674415 & -4.287765 \\
\hline $\mathrm{H}$ & -0.850307 & -4.695771 & -4.300717 \\
\hline $\mathrm{H}$ & 0.023093 & -3.582074 & -5.375475 \\
\hline \multicolumn{4}{|c|}{$3 b^{2+}$} \\
\hline \multicolumn{4}{|c|}{ Singlet } \\
\hline \multicolumn{4}{|c|}{ Total energy, -1918.99593 hartrees } \\
\hline $\mathrm{C}$ & 0.069924 & -0.132700 & 1.498181 \\
\hline $\mathrm{C}$ & 0.069924 & -0.132700 & 0027 \\
\hline $\mathrm{C}$ & 0.091607 & 1.032902 & -0.79167 \\
\hline $\mathrm{C}$ & 0.048241 & -1.298302 & -0.791678 \\
\hline $\mathrm{C}$ & 0.069924 & 2.404022 & -0.46702 \\
\hline $\mathrm{C}$ & 0.069924 & -2.669422 & -0.46702 \\
\hline
\end{tabular}




$\begin{array}{cccc}\mathrm{C} & 0.060853 & 3.342150 & -1.493631 \\ \mathrm{C} & 0.078995 & -3.607550 & -1.493631 \\ \mathrm{C} & 0.060653 & 2.911529 & -2.830258 \\ \mathrm{C} & 0.079195 & -3.176929 & -2.830258 \\ \mathrm{C} & 0.064801 & 1.525572 & -3.178202 \\ \mathrm{C} & 0.075047 & -1.790972 & -3.178202 \\ \mathrm{C} & 0.074461 & 0.608273 & -2.160795 \\ \mathrm{C} & 0.065387 & -0.873673 & -2.160795 \\ \mathrm{C} & 1.086975 & 0.440190 & 2.329879 \\ \mathrm{C} & -0.947127 & -0.705590 & 2.329879 \\ \mathrm{C} & 2.300515 & 1.070255 & 1.992331 \\ \mathrm{C} & -2.160667 & -1.335655 & 1.992331 \\ \mathrm{C} & 3.132704 & 1.508205 & 3.026101 \\ \mathrm{C} & -2.992856 & -1.773605 & 3.026101 \\ \mathrm{C} & 2.760275 & 1.305211 & 4.357068 \\ \mathrm{C} & -2.620427 & -1.570611 & 4.357068 \\ \mathrm{C} & 1.544293 & 0.654782 & 4.707777 \\ \mathrm{C} & -1.404445 & -0.920182 & 4.707777 \\ \mathrm{C} & 0.719914 & 0.223359 & 3.696990 \\ \mathrm{C} & -0.580066 & -0.488759 & 3.696990 \\ \mathrm{H} & 0.056317 & 2.738225 & 0.565706 \\ \mathrm{H} & 0.083531 & -3.003625 & 0.565706 \\ \mathrm{H} & 0.046136 & 4.403820 & -1.274429 \\ \mathrm{H} & 0.093712 & -4.669220 & -1.274429 \\ \mathrm{H} & 0.057950 & 1.241885 & -4.225450 \\ \mathrm{H} & 0.081898 & -1.507285 & -4.225450 \\ \mathrm{H} & 2.592430 & 1.210547 & 0.956113 \\ \mathrm{H} & -2.452582 & -1.475947 & 0.956113 \\ \mathrm{H} & 4.072704 & 1.998315 & 2.796645 \\ \mathrm{H} & -3.932856 & -2.263715 & 2.796645 \\ \mathrm{H} & 3.418097 & 1.645655 & 5.151462 \\ \mathrm{H} & -3.278249 & -1.911055 & 5.151462 \\ \mathrm{H} & 1.295489 & 0.510637 & 5.754575 \\ \mathrm{H} & -1.155641 & -0.776037 & 5.754575 \\ \mathrm{Cl} & 0.094256 & -4.329634 & -4.092990 \\ \mathrm{Cl} & 0.045592 & 4.064234 & -4.092990\end{array}$

Triplet

Total energy, -1918.974398 hartrees

$\begin{array}{cccc}\text { C } & 0.000000 & 0.000000 & 1.514935 \\ \text { C } & 0.000000 & 0.000000 & 0.079645 \\ \text { C } & 0.039924 & 1.160078 & -0.784116 \\ \text { C } & -0.039924 & -1.160078 & -0.784116 \\ \text { C } & 0.000000 & 2.533640 & -0.490573 \\ \text { C } & 0.000000 & -2.533640 & -0.490573 \\ \text { C } & -0.026537 & 3.439490 & -1.539354\end{array}$




$\begin{array}{clll}\mathrm{C} & 0.026537 & -3.439490 & -1.539354 \\ \mathrm{C} & -0.022542 & 2.993054 & -2.901652 \\ \mathrm{C} & 0.022542 & -2.993054 & -2.901652 \\ \mathrm{C} & -0.012503 & 1.629768 & -3.219567 \\ \mathrm{C} & 0.012503 & -1.629768 & -3.219567 \\ \mathrm{C} & 0.010051 & 0.720714 & -2.170534 \\ \mathrm{C} & -0.010051 & -0.720714 & -2.170534 \\ \mathrm{C} & 0.882932 & 0.770025 & 2.364850 \\ \mathrm{C} & -0.882932 & -0.770025 & 2.364850 \\ \mathrm{C} & 1.986674 & 1.583447 & 2.050351 \\ \mathrm{C} & -1.986674 & -1.583447 & 2.050351 \\ \mathrm{C} & 2.730086 & 2.142287 & 3.092768 \\ \mathrm{C} & -2.730086 & -2.142287 & 3.092768 \\ \mathrm{C} & 2.390973 & 1.872193 & 4.421454 \\ \mathrm{C} & -2.390973 & -1.872193 & 4.421454 \\ \mathrm{C} & 1.307753 & 1.021086 & 4.750254 \\ \mathrm{C} & -1.307753 & -1.021086 & 4.750254 \\ \mathrm{C} & 0.567191 & 0.473036 & 3.725504 \\ \mathrm{C} & -0.567191 & -0.473036 & 3.725504 \\ \mathrm{H} & -0.021277 & 2.893001 & 0.532022 \\ \mathrm{H} & 0.021277 & -2.893001 & 0.532022 \\ \mathrm{H} & -0.061287 & 4.506492 & -1.343937 \\ \mathrm{H} & 0.061287 & -4.506492 & -1.343937 \\ \mathrm{H} & -0.032536 & 1.313401 & -4.256964 \\ \mathrm{H} & 0.032536 & -1.313401 & -4.256964 \\ \mathrm{H} & 2.275775 & 1.770899 & 1.021119 \\ \mathrm{H} & -2.275775 & -1.770899 & 1.021119 \\ \mathrm{H} & 3.581203 & 2.777328 & 2.871282 \\ \mathrm{H} & -3.581203 & -2.777328 & 2.871282 \\ \mathrm{H} & 2.980238 & 2.308403 & 5.222493 \\ \mathrm{H} & -2.980238 & -2.308403 & 5.222493 \\ \mathrm{H} & 1.087601 & 0.806506 & 5.791402 \\ \mathrm{H} & -1.087601 & -0.806506 & 5.791402 \\ \mathrm{Cl} & 0.052021 & -4.165411 & -4.142204 \\ \mathrm{Cl} & -0.052021 & 4.165411 & -4.142204 \\ & & & \end{array}$

$\mathbf{3 c}^{\mathbf{2 +}}$
Singlet
Total energy, -1198.27261 hartrees
$\begin{array}{lrrl}\text { C } & 0.156355 & -0.313133 & 1.118132 \\ \text { C } & 0.156355 & -0.313133 & -0.344490 \\ \text { C } & 0.181536 & 0.853075 & -1.171188 \\ \text { C } & 0.131174 & -1.479341 & -1.171188 \\ \text { C } & 0.167175 & 2.224085 & -0.839363 \\ \text { C } & 0.145535 & -2.850351 & -0.839363\end{array}$




$\begin{array}{cccc}\mathrm{C} & 0.163153 & 3.165951 & -1.861919 \\ \mathrm{C} & 0.149557 & -3.792217 & -1.861919 \\ \mathrm{C} & 0.160455 & 2.720178 & -3.187255 \\ \mathrm{C} & 0.152255 & -3.346444 & -3.187255 \\ \mathrm{C} & 0.158405 & 1.346077 & -3.558210 \\ \mathrm{C} & 0.154305 & -1.972343 & -3.558210 \\ \mathrm{C} & 0.163726 & 0.428151 & -2.541779 \\ \mathrm{C} & 0.148984 & -1.054417 & -2.541779 \\ \mathrm{C} & 1.189626 & 0.229976 & 1.948167 \\ \mathrm{C} & -0.876916 & -0.856242 & 1.948167 \\ \mathrm{C} & 2.419134 & 0.828541 & 1.609613 \\ \mathrm{C} & -2.106424 & -1.454807 & 1.609613 \\ \mathrm{C} & 3.262614 & 1.245116 & 2.642869 \\ \mathrm{C} & -2.949904 & -1.871382 & 2.642869 \\ \mathrm{C} & 2.884790 & 1.052997 & 3.974075 \\ \mathrm{C} & -2.572080 & -1.679263 & 3.974075 \\ \mathrm{C} & 1.651518 & 0.435542 & 4.325805 \\ \mathrm{C} & -1.338808 & -1.061808 & 4.325805 \\ \mathrm{C} & 0.816144 & 0.024623 & 3.315762 \\ \mathrm{C} & -0.503434 & -0.650889 & 3.315762 \\ \mathrm{H} & 0.156355 & 2.552102 & 0.195314 \\ \mathrm{H} & 0.156355 & -3.178368 & 0.195314 \\ \mathrm{H} & 0.153683 & 4.231871 & -1.663053 \\ \mathrm{H} & 0.159027 & -4.858137 & -1.663053 \\ \mathrm{H} & 0.150138 & 1.087152 & -4.612202 \\ \mathrm{H} & 0.162572 & -1.713418 & -4.612202 \\ \mathrm{H} & 2.714061 & 0.961554 & 0.573216 \\ \mathrm{H} & -2.401351 & -1.587820 & 0.573216 \\ \mathrm{H} & 4.215034 & 1.710496 & 2.413129 \\ \mathrm{H} & -3.902324 & -2.336762 & 2.413129 \\ \mathrm{H} & 3.551275 & 1.376895 & 4.768193 \\ \mathrm{H} & -3.238565 & -2.003161 & 4.768193 \\ \mathrm{H} & 1.398816 & 0.300469 & 5.372885 \\ \mathrm{H} & -1.086106 & -0.926735 & 5.372885 \\ & 0.158865 & -4.233676 & -4.156432 \\ & 0.153845 & 3.607410 & -4.156432\end{array}$

Triplet

Total energy, -1198.2546595 hartrees
$\begin{array}{llll}\text { C } & 0.000000 & 0.000000 & 1.079652\end{array}$
$\begin{array}{llll}\text { C } & 0.000000 & 0.000000 & -0.358352\end{array}$
$\begin{array}{llll}\text { C } & 0.061786 & 1.168019 & -1.203511\end{array}$
$\begin{array}{lllll}\text { C } & -0.061786 & -1.168019 & -1.203511\end{array}$
$\begin{array}{llll}\text { C } & 0.026604 & 2.541537 & -0.891550\end{array}$
$\begin{array}{llll}\text { C } & -0.026604 & -2.541537 & -0.891550\end{array}$
$\begin{array}{llll}\text { C } & 0.011878 & 3.475135 & -1.922256\end{array}$ 


$\begin{array}{llll}\mathrm{C} & -0.011878 & -3.475135 & -1.922256 \\ \mathrm{C} & 0.007131 & 3.020809 & -3.244244 \\ \mathrm{C} & -0.007131 & -3.020809 & -3.244244 \\ \mathrm{C} & 0.000947 & 1.648526 & -3.596605 \\ \mathrm{C} & -0.000947 & -1.648526 & -3.596605 \\ \mathrm{C} & 0.022783 & 0.738769 & -2.567769 \\ \mathrm{C} & -0.022783 & -0.738769 & -2.567769 \\ \mathrm{C} & 0.899048 & 0.740005 & 1.947098 \\ \mathrm{C} & -0.899048 & -0.740005 & 1.947098 \\ \mathrm{C} & 2.005010 & 1.538052 & 1.669414 \\ \mathrm{C} & -2.005010 & -1.538052 & 1.669414 \\ \mathrm{C} & 2.735080 & 2.069279 & 2.746453 \\ \mathrm{C} & -2.735080 & -2.069279 & 2.746453 \\ \mathrm{C} & 2.381600 & 1.807083 & 4.095365 \\ \mathrm{C} & -2.381600 & -1.807083 & 4.095365 \\ \mathrm{C} & 1.299213 & 0.993717 & 4.396055 \\ \mathrm{C} & -1.299213 & -0.993717 & 4.396055 \\ \mathrm{C} & 0.556162 & 0.444579 & 3.330399 \\ \mathrm{C} & -0.556162 & -0.444579 & 3.330399 \\ \mathrm{H} & 0.000000 & 2.885366 & 0.137097 \\ \mathrm{H} & 0.000000 & -2.885366 & 0.137097 \\ \mathrm{H} & -0.010781 & 4.542061 & -1.730504 \\ \mathrm{H} & 0.010781 & -4.542061 & -1.730504 \\ \mathrm{H} & -0.032907 & 1.373426 & -4.645801 \\ \mathrm{H} & 0.032907 & -1.373426 & -4.645801 \\ \mathrm{H} & 2.317786 & 1.745020 & 0.651859 \\ \mathrm{H} & -2.317786 & -1.745020 & 0.651859 \\ \mathrm{H} & 3.600868 & 2.692396 & 2.542207 \\ \mathrm{H} & -3.600868 & -2.692396 & 2.542207 \\ \mathrm{H} & 2.976676 & 2.240193 & 4.892879 \\ \mathrm{H} & -2.976676 & -2.240193 & 4.892879 \\ \mathrm{H} & 1.039489 & 0.778158 & 5.427690 \\ \mathrm{H} & -1.039489 & -0.778158 & 5.427690 \\ \mathrm{~F} & 0.009970 & -3.904422 & -4.221381 \\ \mathrm{~F} & -0.009970 & 3.904422 & -4.221381\end{array}$


Table S5. HOMO and LUMO energies, in hartrees, for $\mathbf{2}^{\mathbf{2 +}} \mathbf{3}^{\mathbf{2 +}}$

\begin{tabular}{|l|r|r|}
\hline Compound & HOMO energy & LUMO energy \\
\hline $\mathbf{2} \mathbf{a}^{2+}$ & -0.45328 & -0.40428 \\
\hline $\mathbf{2} \mathbf{b}^{2+}$ & -0.46016 & -0.41755 \\
\hline $\mathbf{2} \mathbf{c}^{2+}$ & -0.46812 & -0.41957 \\
\hline $\mathbf{2} \mathbf{d}^{2+}$ & -0.47297 & -0.41074 \\
\hline $\mathbf{3} \mathbf{a}^{2+}$ & -0.45670 & -0.39857 \\
\hline $\mathbf{3}^{2+}$ & -0.46741 & -0.40724 \\
\hline $\mathbf{3} \mathbf{c}^{2+}$ & -0.47442 & -0.41048 \\
\hline
\end{tabular}

Spectrum S1. 3,6 Dimethyl tetrabenzo[5.5]fulvalene, $\mathbf{3 a}^{2+},{ }^{1} \mathrm{H}$ NMR.

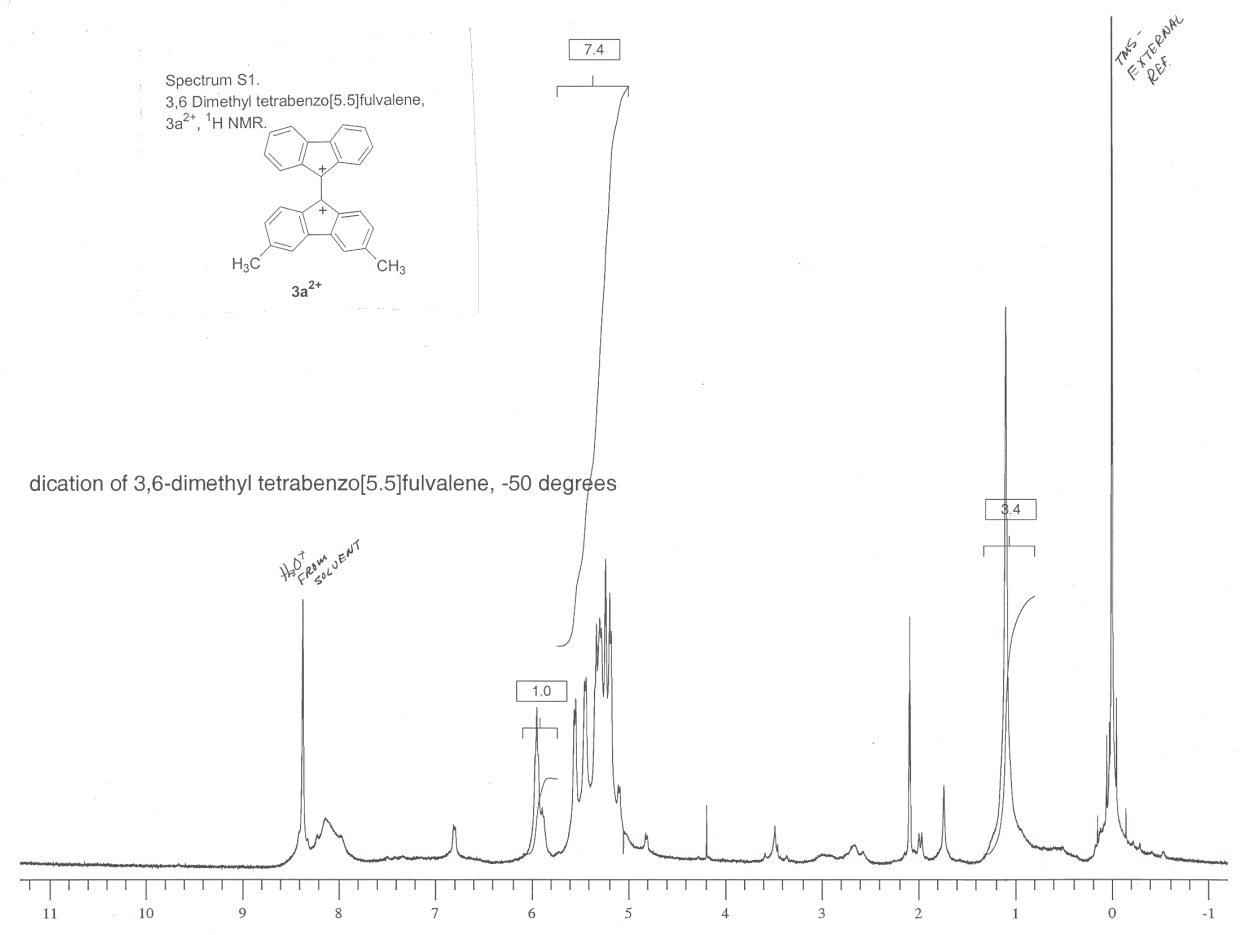




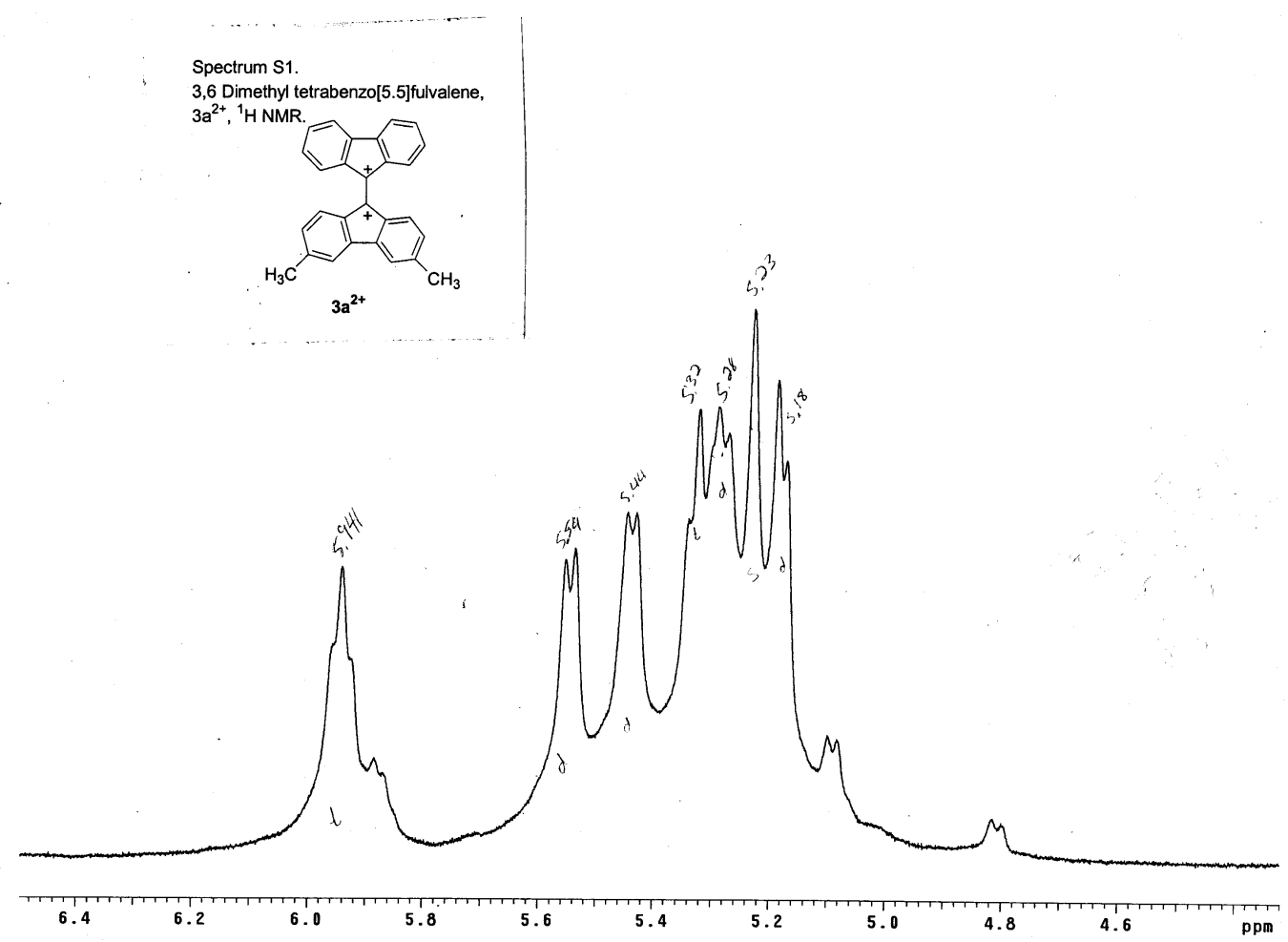

Spectrum S2. 3,6 Dichloro tetrabenzo[5.5]fulvalene, $\mathbf{3 b}^{\mathbf{2 +}},{ }^{1} \mathrm{H}$ NMR.

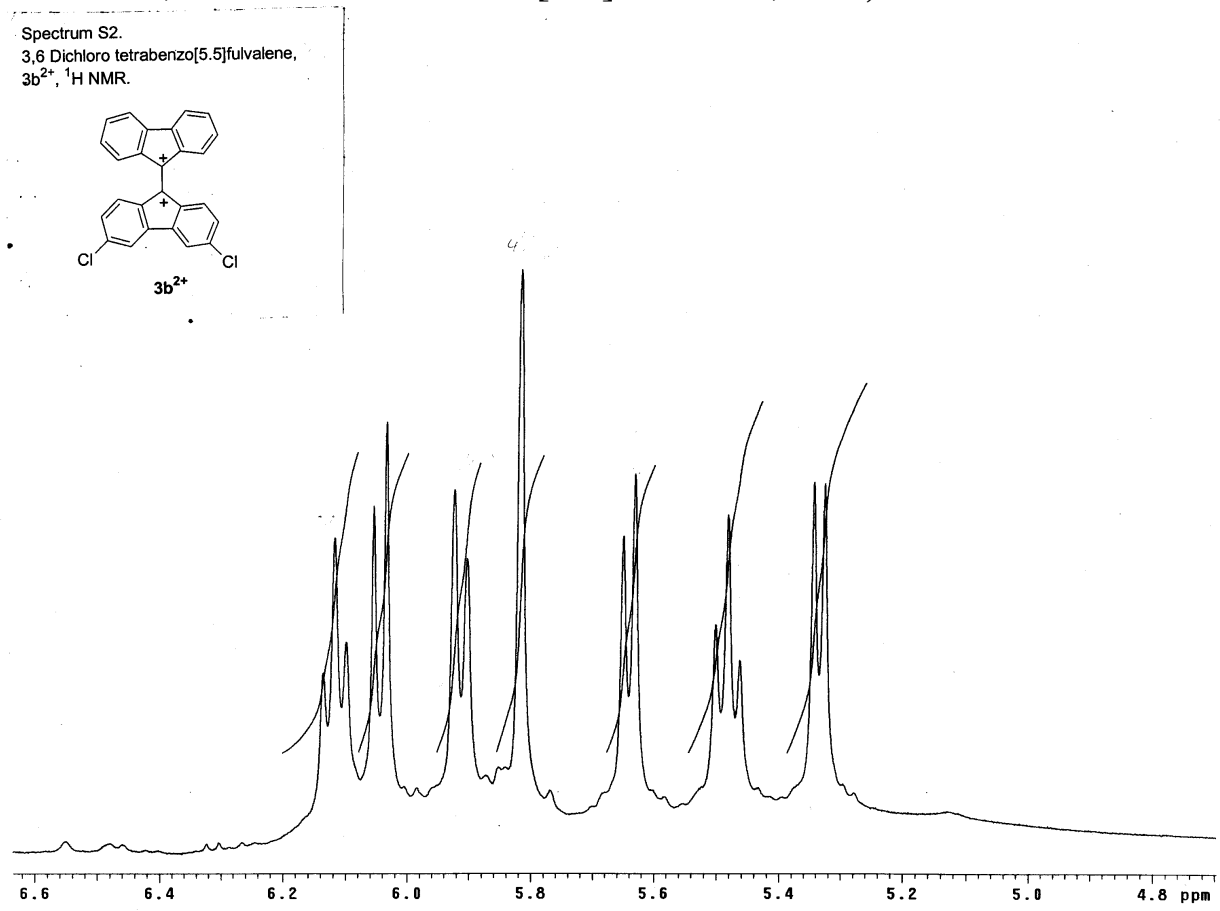

Spectrum S3. 3,6 Dichloro tetrabenzo[5.5]fulvalene, $\mathbf{3} \mathbf{b}^{2+},{ }^{13} \mathrm{C}$ NMR. 


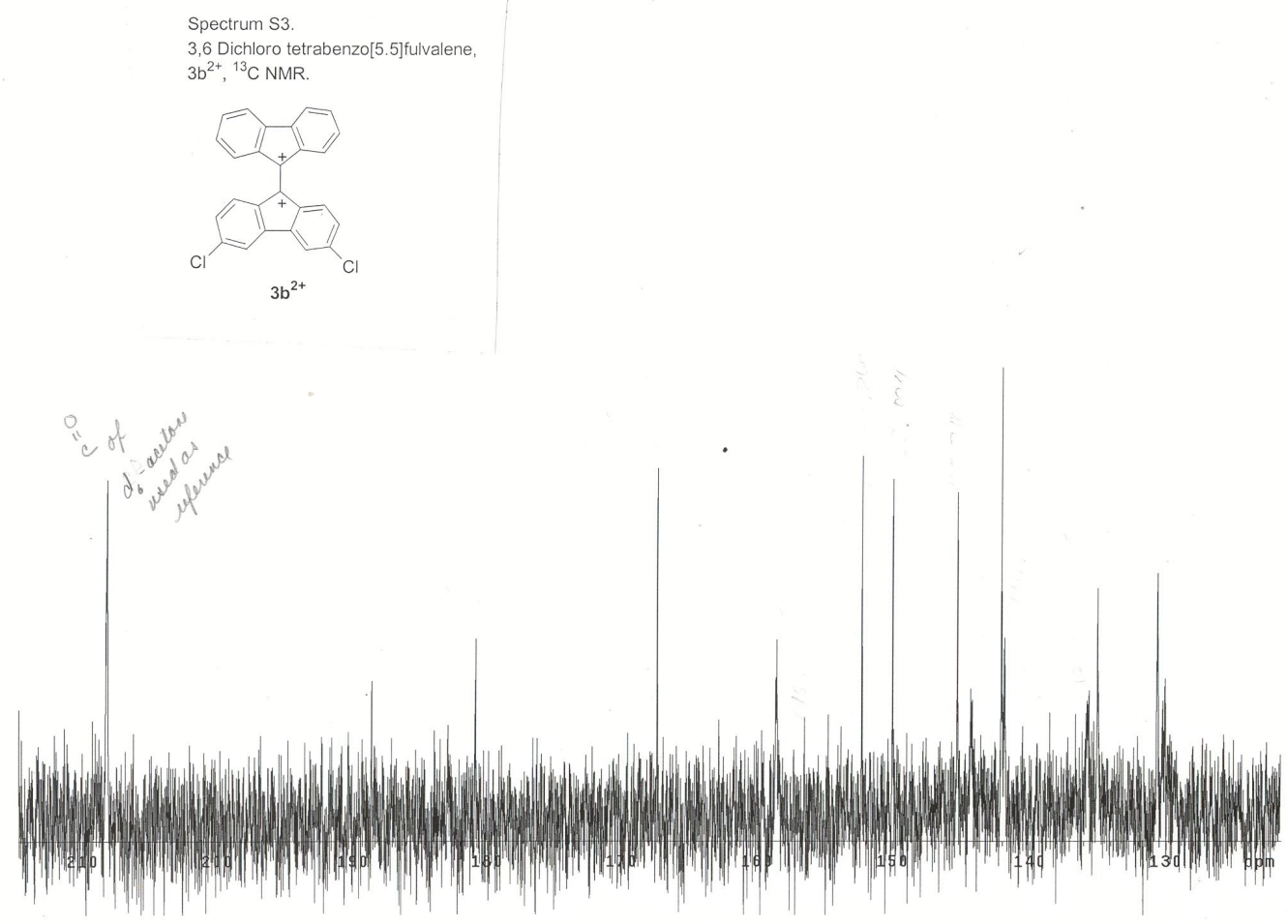


Spectrum S4. 3,6-Difluorotetrabenzo[5.5]fulvalene, $\mathbf{3 c}^{2+},{ }^{1} \mathrm{H}$ NMR.

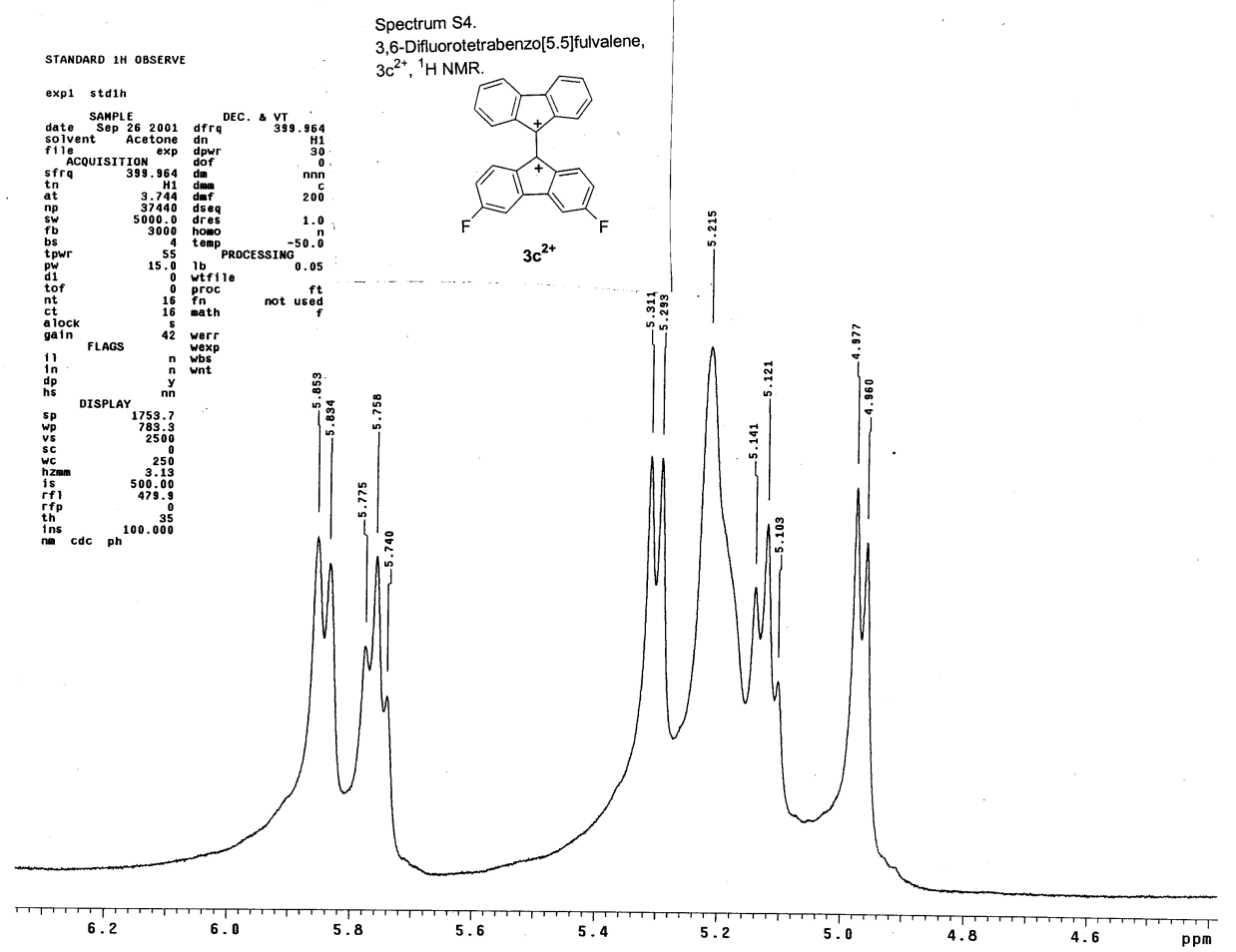

Spectrum S5. 3,6-Difluorotetrabenzo[5.5]fulvalene, $\mathbf{3 c}^{\mathbf{2 +}},{ }^{13} \mathrm{C}$ NMR.

Spectrum S5.

3.6-Difluorotetrabenzo[5.5]fulvalene,

$3 \mathrm{c}^{2+},{ }^{13} \mathrm{C} \mathrm{NMR}$.

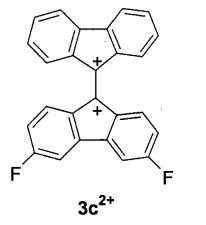

3,6-Difluorotetrabenzo[5.5]fulvalene,

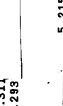

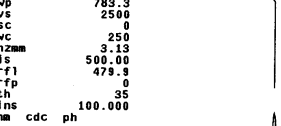


Spectrum S6. 3,6 Dimethyl tetrabenzo[5.5]fulvalene, 3a, ${ }^{1} \mathrm{H}$ NMR.
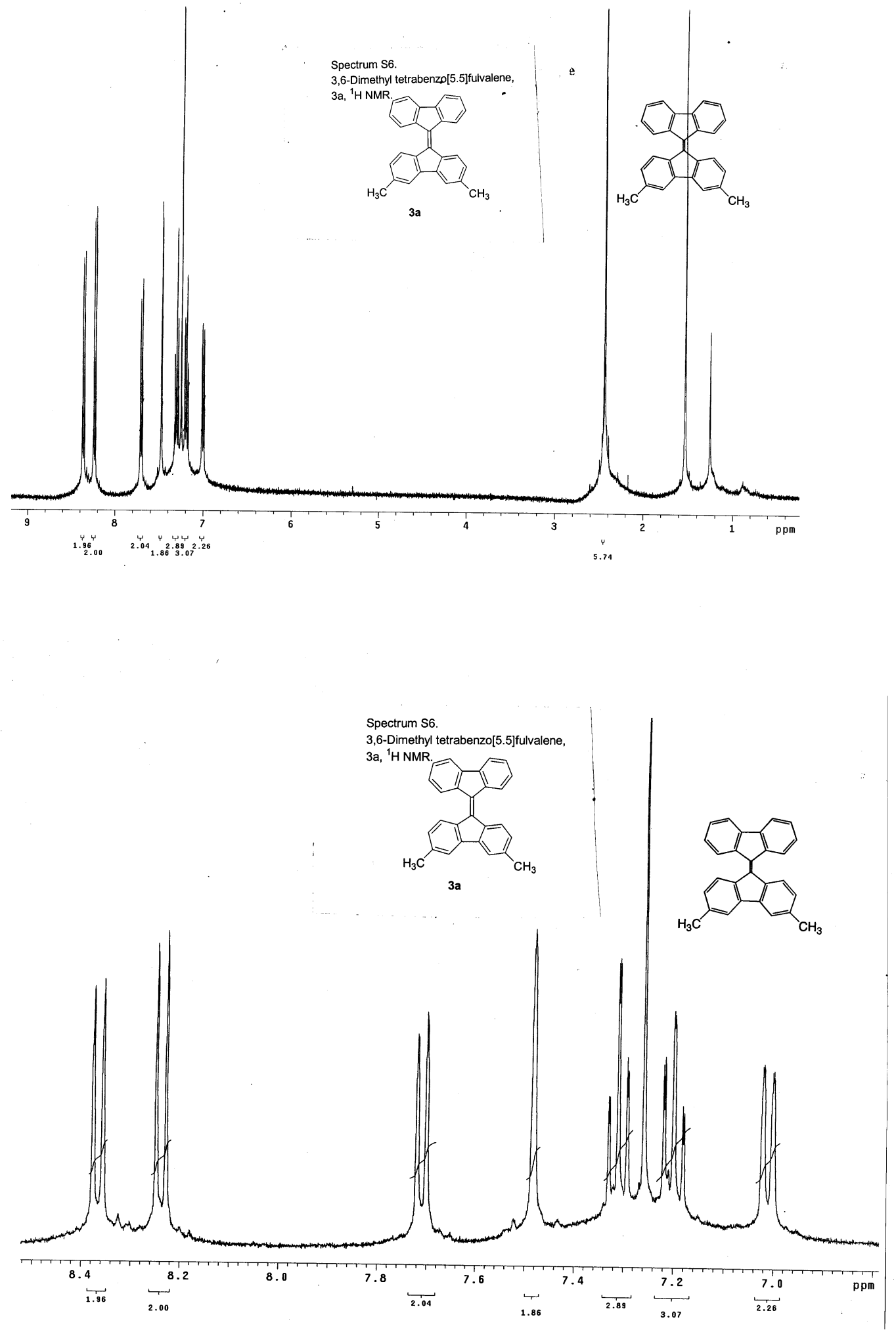
Spectrum S7. 3,6 Dimethyl tetrabenzo[5.5]fulvalene, 3a, ${ }^{13} \mathrm{C}$ NMR.

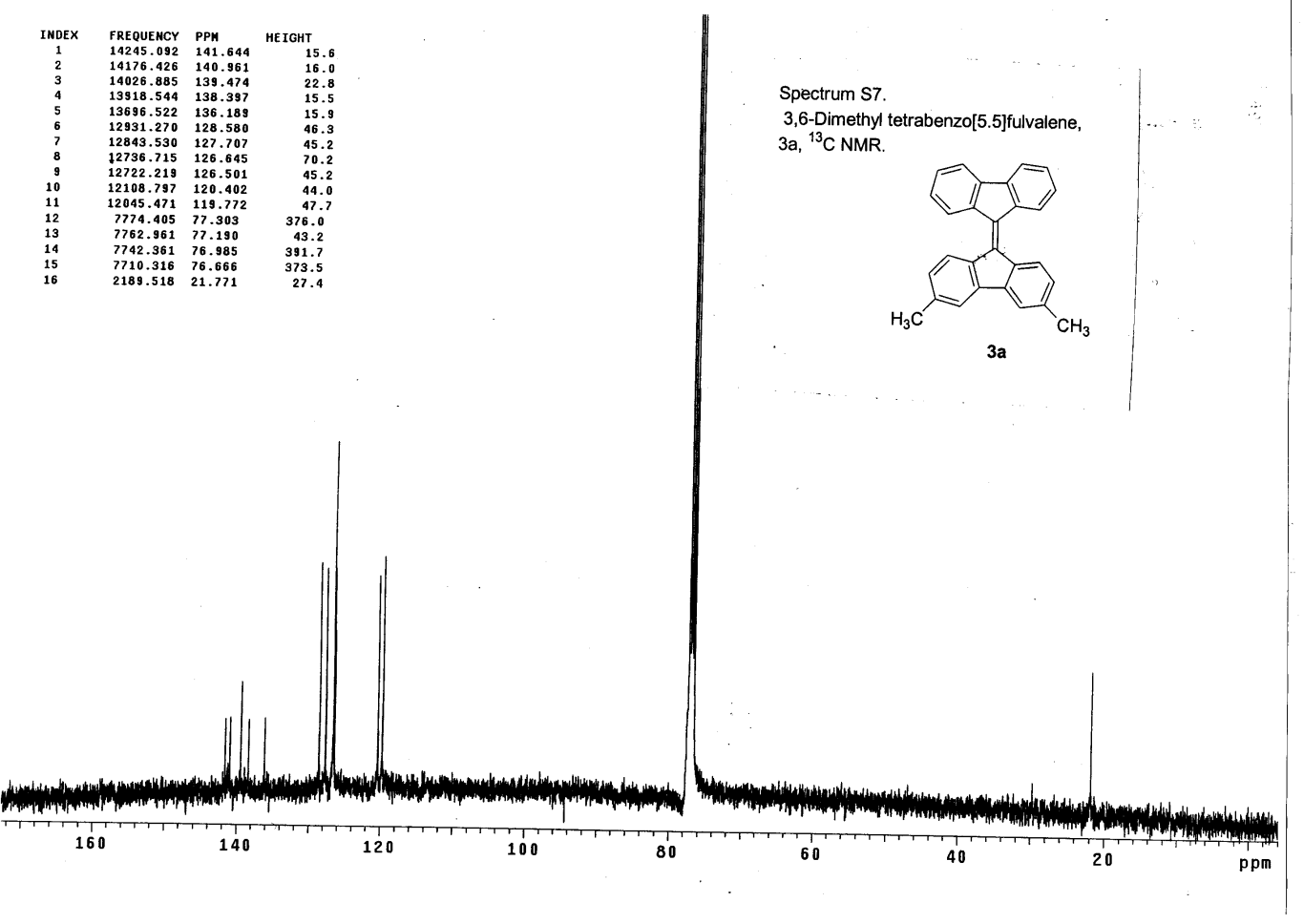


Spectrum S8. 3,6 Dichloro tetrabenzo[5.5]fulvalene, 3b, ${ }^{1} \mathrm{H}$ NMR.
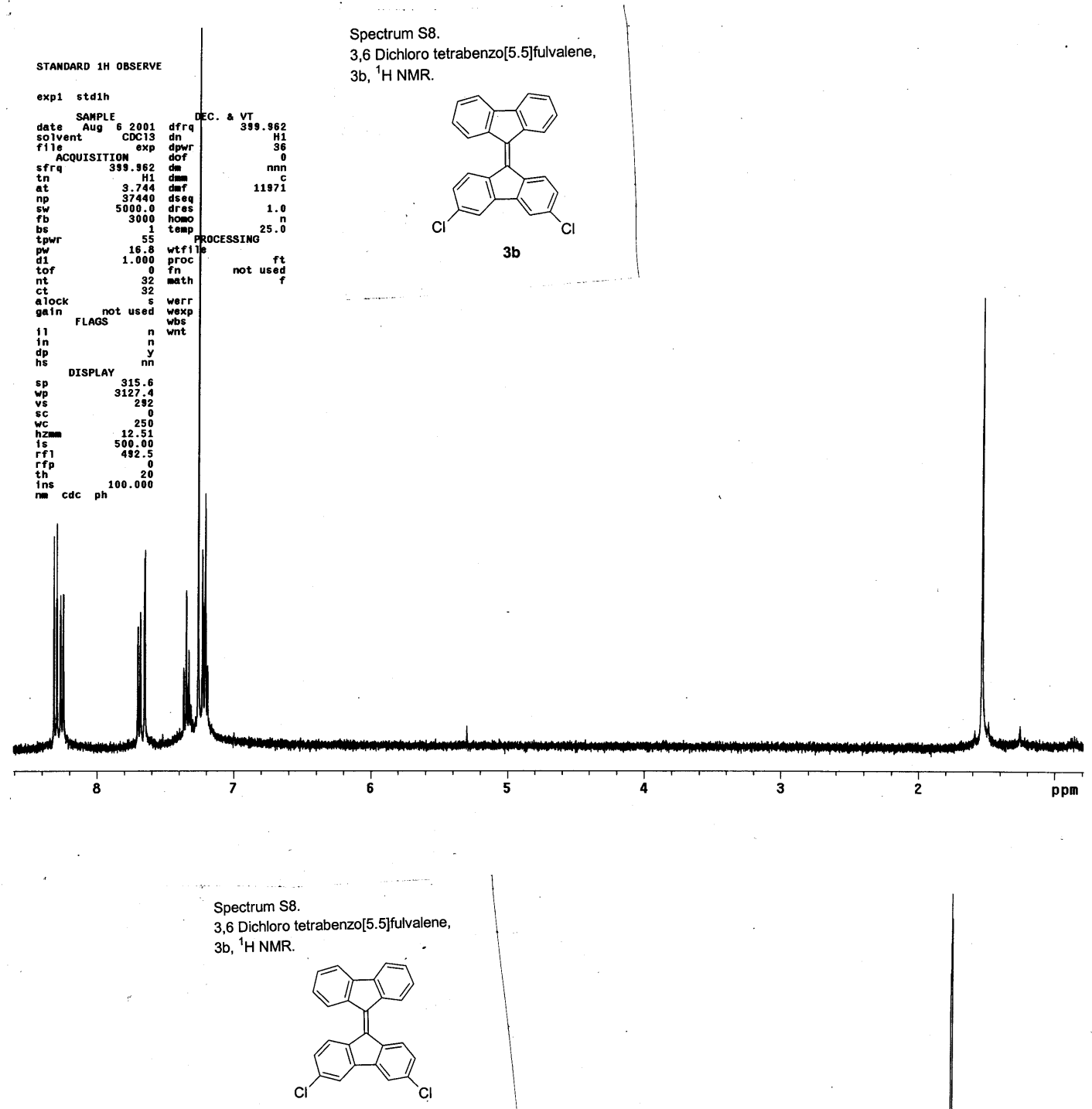

3b

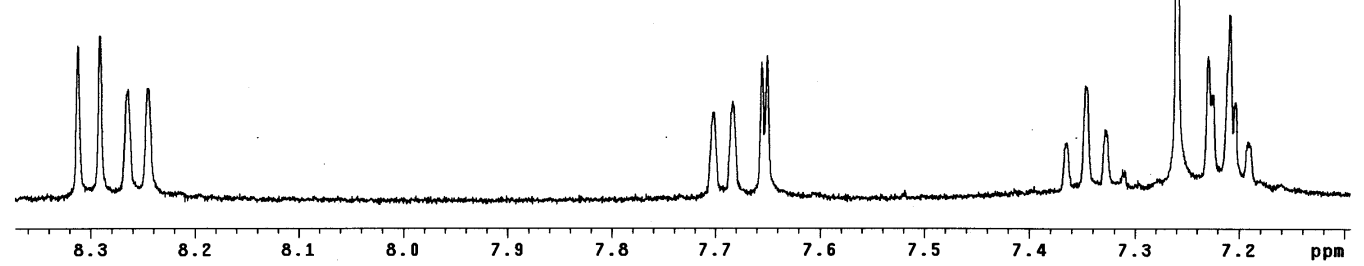


Spectrum S9. 3,6 Dichloro tetrabenzo[5.5]fulvalene, 3b, ${ }^{13} \mathrm{C}$ NMR.

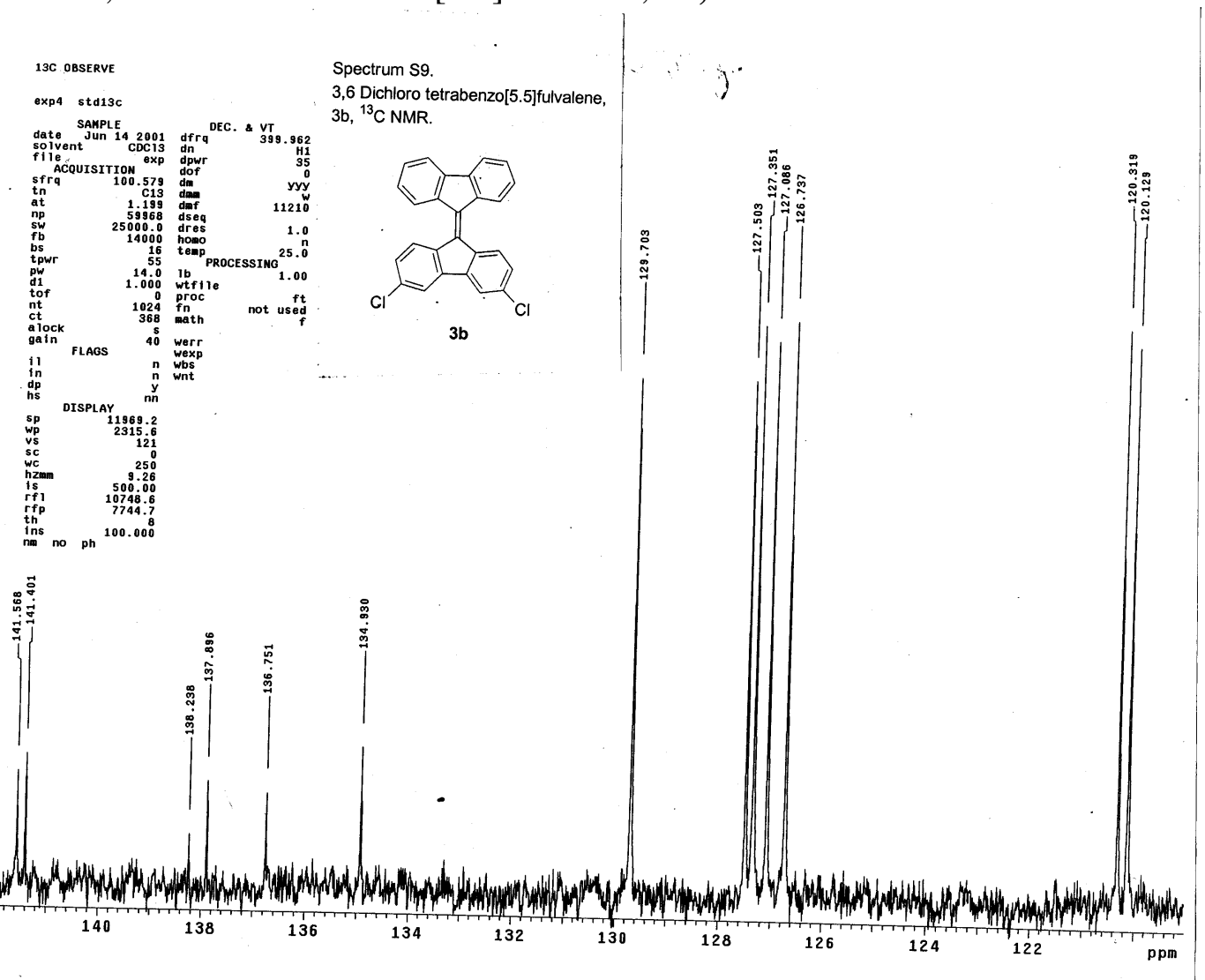


Spectrum S10. 3,6-Difluorotetrabenzo[5.5]fulvalene, 3c, ${ }^{1} \mathrm{H}$ NMR.
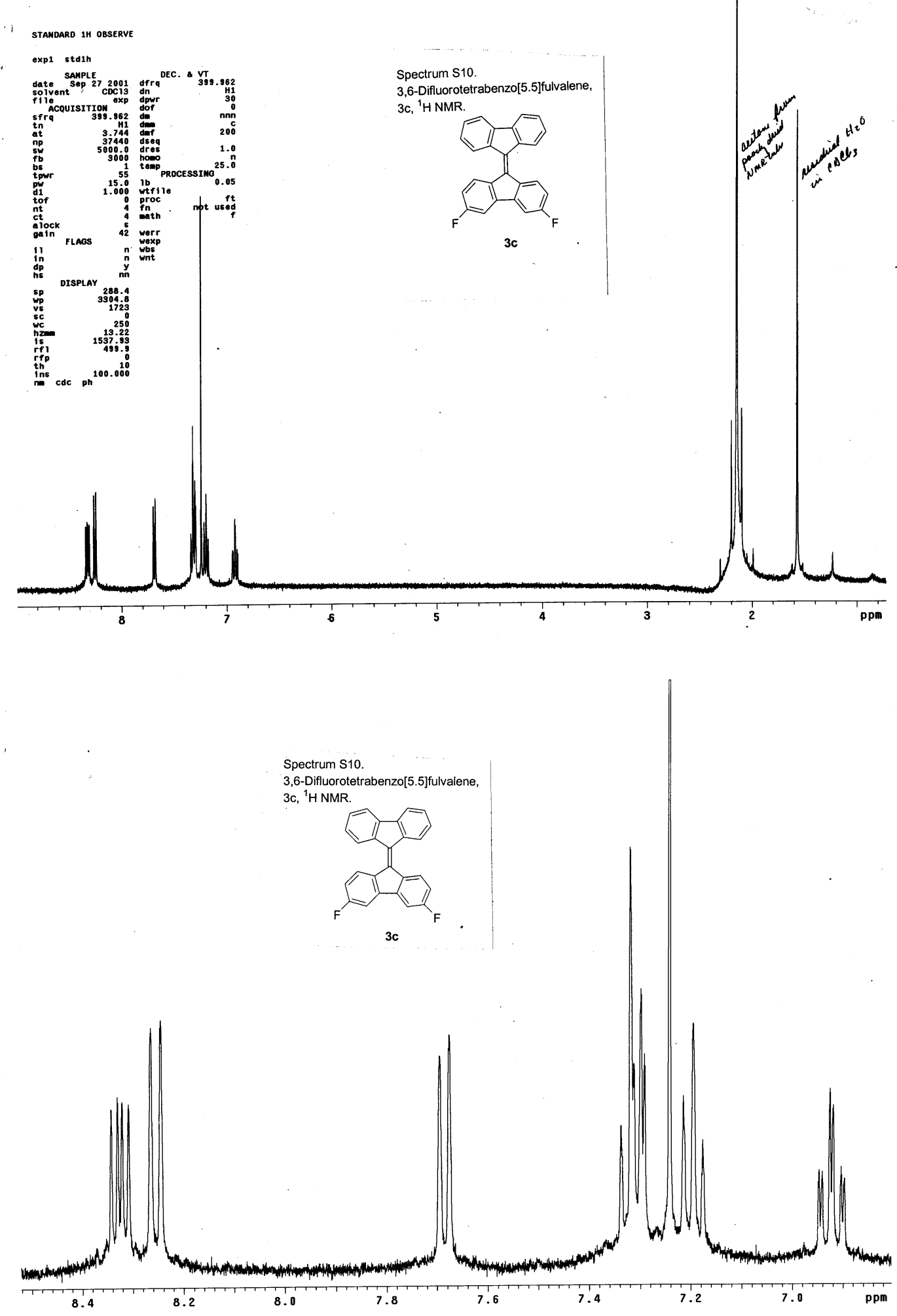
Spectrum S11. 3,6-Difluorotetrabenzo[5.5]fulvalene, 3c, ${ }^{13} \mathrm{C}$ NMR.
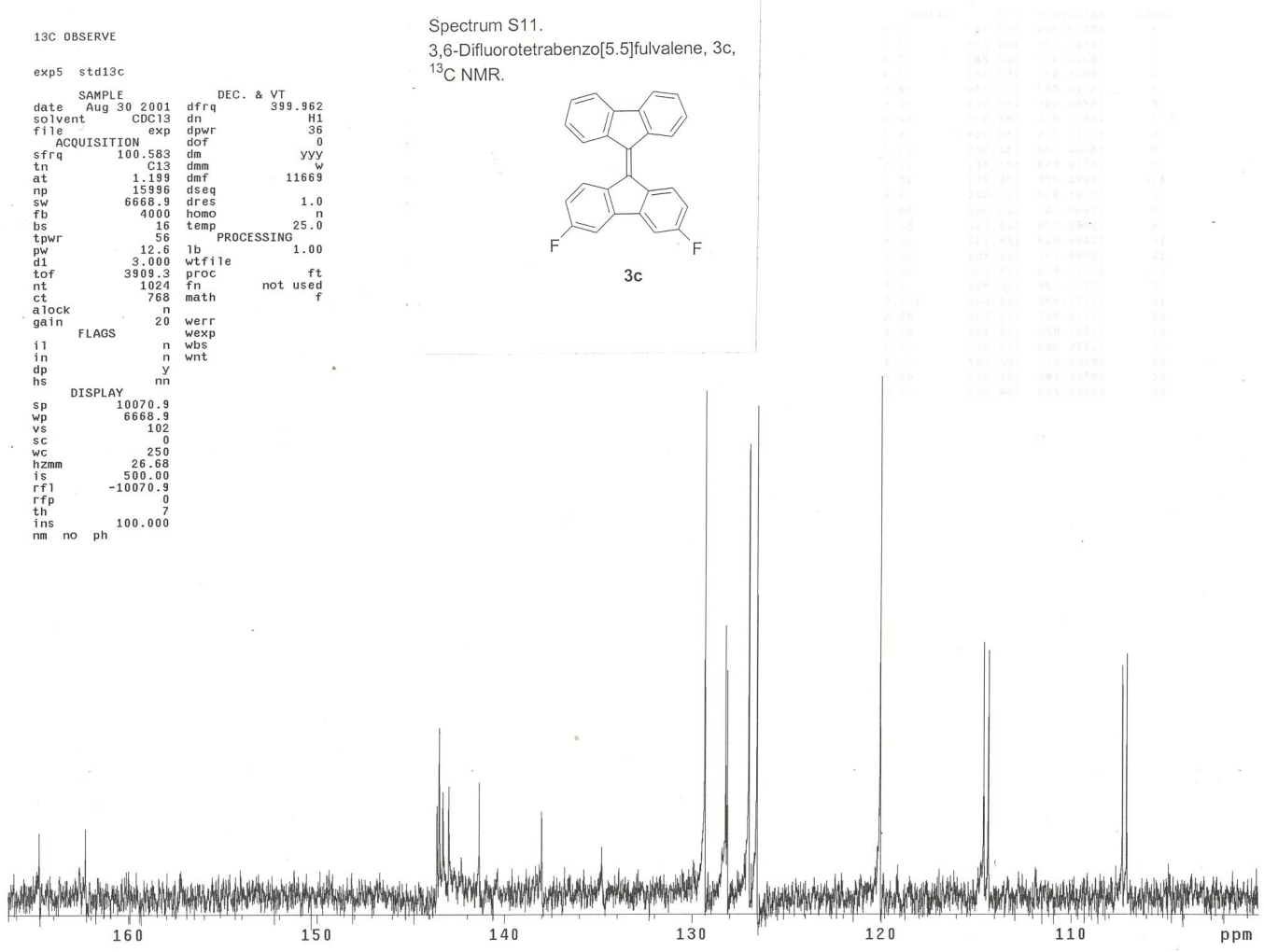


\section{Synthesis of $\mathrm{SO}_{2} \mathrm{ClF}$.}

The $\mathrm{NH}_{4} \mathrm{~F}$ was dried in a drying pistol with $\mathrm{MeOH}$ over night. All glassware was dried in the oven before using.

A $250 \mathrm{~mL}$ 3-necked round bottom flask was equipped with a stir bar, reflux condenser cooled with ice water and topped with a distillation apparatus and a round bottom flask (100ml). The $\mathrm{NH}_{4} \mathrm{~F}$ (50.61 gr, 1.36 mole) was added to the 3-necked flask followed by $\mathrm{SO}_{2} \mathrm{Cl}_{2}(135 \mathrm{~mL}, 1.67$ mole). The reaction mixture was heated with a water bath at $65-70{ }^{\circ} \mathrm{C}$ and refluxed gently and stirred for 3-4h or till there was no more solvent in the 3-necked round bottom flask. A yellow liquid was collected in the receiver at $-78{ }^{\circ} \mathrm{C}$. The yellow liquid was stored in the freezer overnight. Fractional distillation was performed in a closed system. The pot was warmed to no more than till $25-35{ }^{\circ} \mathrm{C}$. The first fraction was discarded. To the yellowish liquid which was collected, $2 \mathrm{~g} \mathrm{SbF}_{5}$ was added to complex $\mathrm{SO}_{2}$, giving a precipitate. The receiving flask was stored overnight in the freezer. Two more fractional distillation were performed the next day, one from $\mathrm{SbF}_{5}$.

$42.93 \mathrm{~g}$ of colorless liquid were obtained, yield $27 \%$. 